OPEN ACCESS

Edited by:

Beiyan Nan,

University of California, Berkeley, USA

Reviewed by:

Deborah Beth Zamble,

University of Toronto, Canada

Andrzej M. Krezel,

Washington University School of

Medicine, USA

*Correspondence:

D. Scott Merrell,

Department of Microbiology and

Immunology, Uniformed Services

University of the Health Sciences,

4301 Jones Bridge Road, Bethesda,

MD 20814, USA

douglas.merrell@usuhs.edu;

Sarah L. J. Michel,

Department of Pharmaceutical

Sciences, School of Pharmacy,

University of Maryland, 20 Penn

Street, Baltimore, MD 21201, USA

smiche/@rx.umaryland.edu

${ }^{\dagger}$ These authors have contributed equally to this work.

Specialty section:

This article was submitted to

Food Microbiology,

a section of the journal

Frontiers in Microbiology

Received: 26 March 2015

Accepted: 20 May 2015

Published: 12 June 2015

Citation:

Carpenter BM, West AL, Gancz H, Servetas SL, Pich OQ, Gillbreath JJ, Hallinger DR, Forsyth $M H$, Merrell DS

and Michel SLJ (2015) Crosstalk

between the HpArsRS

two-component system and HpNikR

is necessary for maximal activation of

urease transcription

Front. Microbiol. 6:558.

doi: 10.3389/fmicb.2015.00558

\section{Crosstalk between the HpArsRS} two-component system and $H p N i k R$ is necessary for maximal activation of urease transcription

\author{
Beth M. Carpenter ${ }^{1+}$, Abby L. West ${ }^{2 \dagger}$, Hanan Gancz ${ }^{1}$, Stephanie L. Servetas ${ }^{1}$, \\ Oscar Q. Pich ${ }^{1}$, Jeremy J. Gilbreath ${ }^{1}$, Daniel R. Hallinger ${ }^{3}$, Mark H. Forsyth ${ }^{3}$, \\ D. Scott Merrell ${ }^{1 *}$ and Sarah L. J. Michel ${ }^{2 *}$ \\ ${ }^{1}$ Department of Microbiology and Immunology, Uniformed Services University of the Health Sciences, Bethesda, MD, USA, \\ ${ }^{2}$ Department of Pharmaceutical Sciences, School of Pharmacy, University of Maryland, Baltimore, Maryland, USA, \\ ${ }^{3}$ Department of Biology, The College of William and Mary, Williamsburg, VA, USA
}

Helicobacter pylori NikR (HpNikR) is a nickel dependent transcription factor that directly regulates a number of genes in this important gastric pathogen. One key gene that is regulated by $H p N i k R$ is ureA, which encodes for the urease enzyme. In vitro DNA binding studies of $H p N i k R$ with the ureA promoter $\left(P_{\text {ureA }}\right)$ previously identified a recognition site that is required for high affinity protein/DNA binding. As a means to determine the in vivo significance of this recognition site and to identify the key DNA sequence determinants required for ure $A$ transcription, herein, we have translated these in vitro results to analysis directly within $H$. pylori. Using a series of GFP reporter constructs in which the $P_{\text {ureA }}$ DNA target was altered, in combination with mutant $H$. pylori strains deficient in key regulatory proteins, we confirmed the importance of the previously identified $H p N i k R$ recognition sequence for $H p N i k R$-dependent ureA transcription. Moreover, we identified a second factor, the HpArsRS two-component system that was required for maximum transcription of ureA. While HpArsRS is known to regulate ureA in response to acid shock, it was previously thought to function independently of $H p N i k R$ and to have no role at neutral $\mathrm{pH}$. However, our qPCR analysis of ureA expression in wildtype, $\Delta$ nikR and $\Delta$ ars $S$ single mutants as well as a $\Delta a r s S / n i k R$ double mutant strain background showed reduced basal level expression of ureA when arsS was absent. Additionally, we determined that both HpNikR and HpArsRS were necessary for maximal expression of ureA under nickel, low $\mathrm{pH}$ and combined nickel and low $\mathrm{pH}$ stresses. In vitro studies of HpArsR-P with the $P_{\text {ureA }}$ DNA target using florescence anisotropy confirmed a direct protein/DNA binding interaction. Together, these data support a model in which $H p A r s R S$ and $H p N i k R$ cooperatively interact to regulate $u r e A$ transcription under various environmental conditions. This is the first time that direct "cross-talk" between HpArsRS and $H p N i k R$ at neutral $\mathrm{pH}$ has been demonstrated.

Keywords: nikR, arsRS, pylori, urease, Helicobacter, regulation, pH 


\section{Introduction}

Helicobacter pylori is a microaerophilic, Gram negative pathogen that infects over half of the world's human population (Marshall and Warren, 1984; Loughlin, 2003). Colonization occurs in the highly acidic gastric mucosal layer as well as at the gastric epithelial surface of the human stomach. Prolonged $H$. pylori infection is associated with the development of gastritis, peptic ulcer disease, Mucosal-Associated Lymphoid Tissue (MALT) lymphoma and gastric adenocarcinoma (Marshall and Warren, 1984; Cover and Blaser, 1992; Sepulveda and Coelho, 2002; Loughlin, 2003; Kusters et al., 2006). Current therapies for $H$. pylori infection require antibiotic cocktails of two, three or four drugs that are often not well tolerated due to adverse side effects (Loughlin, 2003; Kusters et al., 2006). If $H$. pylori infection is left untreated, colonization will persist throughout a person's lifetime (Marshall and Warren, 1984; Blaser, 1990; Cover and Blaser, 1992; Dunn et al., 1997; Sepulveda and Coelho, 2002; Loughlin, 2003). The propensity for chronic infection by $H$. pylori, coupled with the large rate of infection, manifests as a significant disease burden worldwide (Kusters et al., 2006). Thus, there is a great need to develop novel targeted anti-Helicobacter agents that are well tolerated (Loughlin, 2003).

While it displays optimal growth at neutral $\mathrm{pH}, H$. pylori is one of a select number of bacteria that can survive under highly acidic conditions; this makes it ideally suited to life in the gastric mucosa. The cytosolic $\mathrm{pH}$ of $H$. pylori ranges from 5.3 to 7.5, and the organism can endure periodic acid shocks of $\mathrm{pH}<2$ (Wen et al., 2003, 2009; van Vliet et al., 2004a). One key feature that enables $H$. pylori to survive under such harsh conditions is its ability to convert host urea into ammonia, which serves to buffer the cytoplasmic/periplasmic $\mathrm{pH}$, as well as to neutralize the immediate environment upon excretion from the bacterial cell (Wen et al., 2003; van Vliet et al., 2004a,b; Sachs et al., 2005, 2006; Scott et al., 2007). The majority of ammonia is produced by the nickel dependent urease enzyme, which converts urea to ammonia and bicarbonate. Urease accounts for approximately $10 \%$ of the total protein content of $H$. pylori (van Vliet et al., 2004a; Carter et al., 2009), and expression of the operon of genes that encode urease is known to be subject to environmental regulation.

The nickel dependent metalloregulatory protein, $H p N i k R$, is known to be a key regulator of urease expression (van Vliet et al., 2002; Contreras et al., 2003; Ernst et al., 2005; Dosanjh and Michel, 2006; Maier et al., 2007). HpNikR functions as a tetrameric metalloregulatory protein. Each $H p \mathrm{NikR}$ monomer is comprised of two domains, named the N-terminal and Cterminal domains (West et al., 2010, 2012; Benini et al., 2011). To form the functional tetrameric protein, all four C-terminal domains form a central tetrameric metal binding domain (MBD), which serves as the site of nickel coordination. The MBD is then flanked on either side by two $\mathrm{N}$-terminal domains that fold to form two dimeric DNA binding domains (DBD). The DBDs adopt a classic ribbon-helix-helix fold, which is commonly found in DNA binding proteins (Figure 1A) (Chivers and Sauer, 1999, 2000, 2002; Chivers and Tahirov, 2005; Schreiter and Drennan, 2007; West et al., 2010).
$H p \mathrm{NikR}$ requires $\mathrm{Ni}(\mathrm{II})$ coordination to the $\mathrm{MBD}$ in order to recognize and bind to DNA via the DBD (Abraham et al., 2006; Benanti and Chivers, 2007; Dosanjh et al., 2007; Bahlawane et al., 2010). Crystallographic studies conducted by one of our laboratories have shown that $\mathrm{Ni}$ (II) coordinates to two distinct sites at the MBD of $H p N i k R$ : two $\mathrm{Ni}(\mathrm{II})$ ions coordinate at square planar sites that utilize a $3 \mathrm{His} / 1 \mathrm{Cys}$ ligand set and two $\mathrm{Ni}$ (II) ions coordinate to a square pyramidal/octahedral sites that utilize a $3 \mathrm{His} / 2-3 \mathrm{H}_{2} \mathrm{O}$ ligand set (Figure 1A) (West et al., 2010, 2012). This heterogeneous nickel coordination controls the overall flexibility of the DBDs to favor high affinity DNA binding (West et al., 2012).

$H p \mathrm{NikR}$ is a global regulator of transcription in $H$. pylori (Contreras et al., 2003; van Vliet et al., 2004a; Ernst et al., 2005; Abraham et al., 2006). In addition to urease, $H p$ NikR activates and represses the expression of at least forty genes in response to nickel availability (van Vliet et al., 2002; Contreras et al., 2003; Ernst et al., 2005, 2006; Abraham et al., 2006; Davis et al., 2006; Dosanjh and Michel, 2006; Benanti and Chivers, 2007, 2011; Dosanjh et al., 2007, 2009; Zambelli et al., 2008; Li and Zamble, 2009; Muller et al., 2011; Romagnoli et al., 2011; Evans and Michel, 2012; Jones et al., 2015). Genes which have been shown to be directly regulated by $H p N i k R$ encode for proteins that regulate nickel uptake (nix $A$, frpB4, fecA3, frpB2, ceuE), storage ( $h p n, h p n$-like) and regulation (nikR), genes involved in iron uptake $(e x b B)$ and regulation (fur), genes involved in infection $(h s p A)$, and genes that encode for outer membrane proteins (exbB/tonB) (van Vliet et al., 2002; Contreras et al., 2003; Delany et al., 2005; Ernst et al., 2005, 2006; Abraham et al., 2006; Davis et al., 2006; Benanti and Chivers, 2007; Dosanjh et al., 2007, 2009; Jones et al., 2015). The direct binding of $H p N i k R$ to the promoter sequences of these genes has been shown via DNase footprinting, electrophoretic mobility shift assays (EMSA), fluorescence anisotropy (FA) and/or isothermal titration calorimetry (ITC) (Contreras et al., 2003; Delany et al., 2005; Ernst et al., 2005, 2006; Abraham et al., 2006; Davis et al., 2006; Benanti and Chivers, 2007, 2011; Dosanjh et al., 2007, 2009; Zambelli et al., 2008; Li and Zamble, 2009; Muller et al., 2011; Romagnoli et al., 2011; Jones et al., 2015). In addition, Krezel and co-workers recently reported a direct role for the RNA polymerase alpha-subunit C-terminal domain in promoting $H p N i k R / u r e A$ binding (Borin et al., 2014). From those studies, a general model for $H p N i k R$ gene regulation has been formulated. In this model, $H p \mathrm{NikR}$ activates transcription by binding upstream of the RNA polymerase binding site, which aids in the recruitment of RNA polymerase (i.e., for ureA). In contrast, $H p N i k R$ represses transcription by a simple steric hindrance mechanism in which $H p$ NikR blocks the interaction of RNA polymerase with the promoter by binding at promoter sequences overlapping the -10 or -35 hexameric boxes (e.g., nikR, nixA, fur, frpB4, exbB, fecA3) (van Vliet et al., 2002; Delany et al., 2005; Ernst et al., 2006; Wolfram et al., 2006; Danielli et al., 2009; Dosanjh et al., 2009). In addition to the genes for which direct protein/DNA binding has been established, approximately 30 additional genes have been predicted to be regulated by $H p N i k R$ (Contreras et al., 2003; Delany et al., 2005; Ernst et al., 2005; Abraham et al., 2006; Davis et al., 2006; Ernst et al., 2006; Benanti and Chivers, 2007; Dosanjh et al., 2007; Zambelli et al., 2008; 


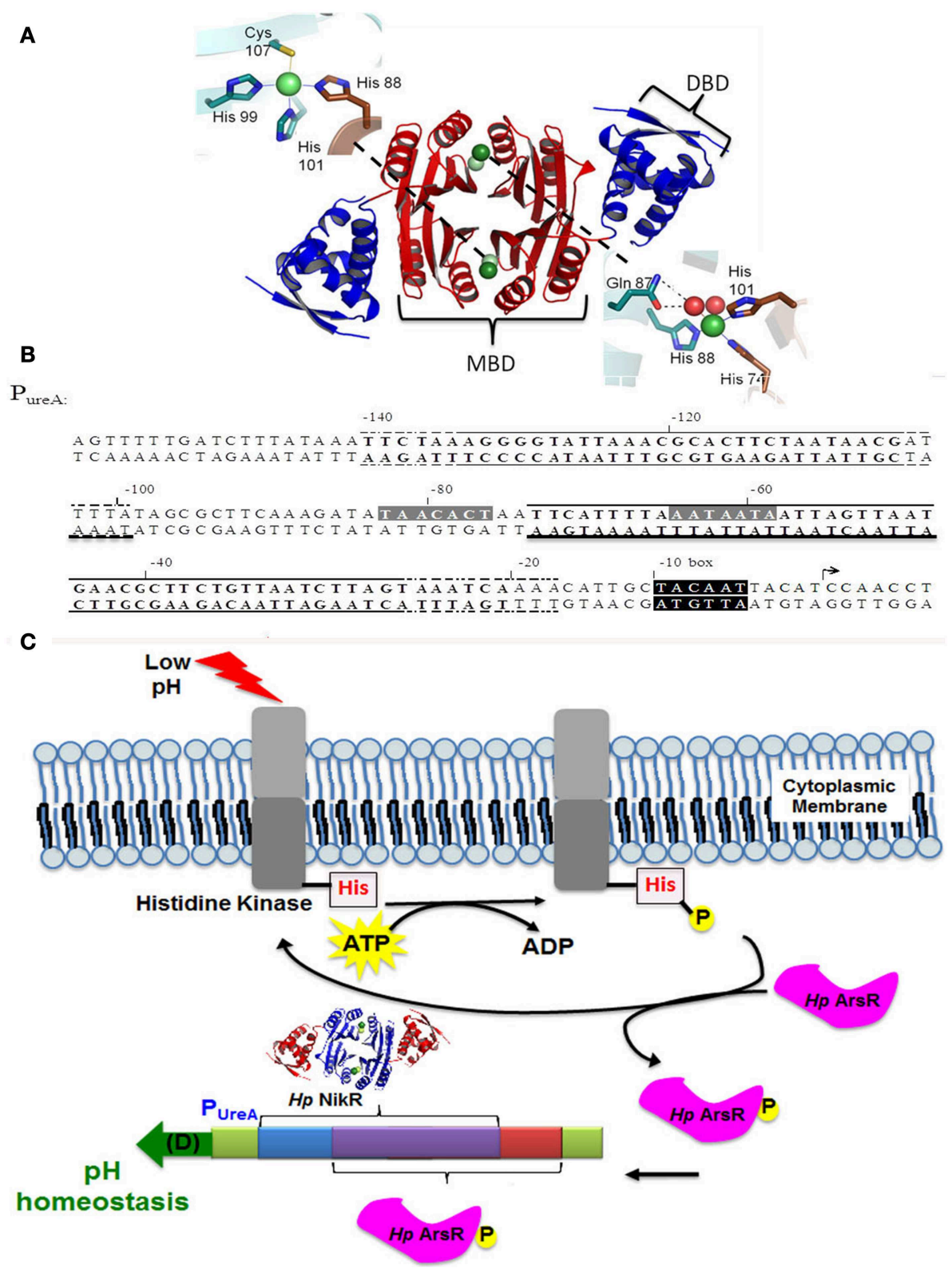

FIGURE 1 | Structure of holo-HpNikR and architecture of the ureA promoter. (A) The structure of holo-HpNikR. Essential areas of the protein are highlighted as follows: the metal binding domain (MBD) in red, the DNA binding domain (DBD) in blue, 4-site (top left) and 5/6-site (bottom right). This image was constructed in pymol (accession number pdb $3 L G H)$. (B) The recognition sites for HpNikR and HpArsR on the $H$. pylori ureA promoter. Highlighted in gray is the recognition site for
HpNikR, while solid and dashed lines above the sequence designate the minimum and maximum protected regions from DNase protection assays for the two distinct binding sites of HpArsR as previously described (Pflock et al., 2005). (C) A cartoon demonstrating the operator overlap at the ureA promoter for HpNikR (pdb 3LGH) and HpArsR within the context of the biological role of HpArsR. The region colored purple represents the overlapping promoter sites.
Dosanjh et al., 2009; Li and Zamble, 2009; Benanti and Chivers, 2011; Muller et al., 2011; Romagnoli et al., 2011). Whether these additional genes are regulated directly or indirectly has yet to be established. These genes include components involved in motility (cheV, flaA, flaB) and stress response ( $h r c A, \operatorname{grp} E, d n a K)$, as well as outer membrane proteins (omp11, omp31, omp32) (Contreras et al., 2003; Dosanjh et al., 2009).
The promoters for the genes that are directly regulated by $H p N i k R$ share common sequences, but are not identical. These DNA targets share a partially palindromic sequence composed of two sets of seven base pairs (half-sites), separated by eleven bases (Delany et al., 2005; Dosanjh et al., 2007, 2009; Stoof et al., 2010; Evans and Michel, 2012). HpNikR binds to a subset of the promoters with high affinity $\left(K_{d} \sim \mathrm{nM}\right)$ and a subset of the 
promoters with low affinity ( $\left.K_{d} \sim \mu \mathrm{M}\right)$ (Dosanjh et al., 2009). The promoter sequences for which high affinity recognition is measured are from genes that encode for nickel regulated proteins (ureA, nixA, frpb4, fecA3), while the promoter sequences for which low affinity binding has been measured are from genes that encode for other proteins (fur, nikR, exbB) (Dosanjh et al., 2009). Thus, in vitro HpNikR preferentially recognizes genes that encode for nickel-regulated proteins. The DNA target sequences for which high affinity DNA binding is observed have greater conservation of sequence at the second half-site (Evans and Michel, 2012). Studies using the ureA promoter in which the DNA sequences have been systematically altered have identified key bases within the second half-site that are essential for highaffinity protein/DNA binding (Delany et al., 2005; Dosanjh et al., 2009; Evans and Michel, 2012). In addition, the intact partial-palindrome is required for high affinity DNA binding by $H p$ NikR in vitro (Dosanjh et al., 2009). When either half of the partial palindrome was modified to all cytosines, the affinity of $H p$ NikR for the ureA promoter decreased from $8.0 \pm 1 \mathrm{nM}$ to $1000 \pm 94 \mathrm{nM}$ for WT/C (CTTCAAAGATATAACACTAATTC ATTTTACCCCCCCATTAGTTAATGA) and $4900 \pm 780 \mathrm{nM}$ for C/WT (CTTCAAAGATACCCCCCCAATTCATTTTAAAT AATAATTAGTTAATGA) (Dosanjh et al., 2009; West et al., 2012). When both halves of the palindrome were modified, DNA binding was fully abrogated (Dosanjh et al., 2009).

The HpArsRS two-component system has also been shown to regulate a wide variety of genes in $H$. pylori. These genes include those that encode for proteins involved in acid resistance, (including urease), acetone metabolism (acetone carboxylase), resistance to oxidative stress (thioredoxin reductase), and quorum sensing (Pflock et al., 2005, 2006a,b; Wen et al., 2007; Goodwin et al., 2008; Loh et al., 2010). Within the regulatory pathway, $H p A r s S$ serves as a sensor protein that phosphorylates the $H p A r s R$ response regulator. Phosphorylation occurs via a two-step process: HpArsS autophosphorylates at histidine 214 and then transfers the phosphate to aspartic acid 52 on HpArsR to generate HpArsR-P (Schar et al., 2005; Pflock et al., 2006a; Joseph and Beier, 2007; Gupta et al., 2009; Muller et al., 2009). HpArsR appears essential for bacterial viability and binds to different promoter elements based on the phosporylation state. Binding at $P_{\text {ure }}$ requires phosphorylation and the binding site of $H p$ ArsR-P overlaps with the binding site recognized by $H p N i k R$ (Pflock et al., 2005) (Figures 1B,C). Given that HpArsR and $H p$ NikR respond to different environmental conditions, acid and nickel, respectively, these two regulators are believed to function independently of one another (Pflock et al., 2005).

Most of the studies that have investigated binding of $H p N i k R$ to target promoters have been conducted in vitro. However, it is clear that the cytoplasm of the bacterial cell is a much more complex environment than the one modeled in an in vitro experiment. There is one in vivo study of $H p N i k R$ mediated nickel response in which quantitative real-time PCR was used to measure $H p N i k R$ regulation of target genes (Muller et al., 2011). In this study, a complex relationship between $\mathrm{Ni}(\mathrm{II})$ availability and activation or repression of a series of genes was determined, suggesting that $H p N i k R$ activity in vivo may be more complex than observed in vitro. The goal of the studies described herein was to determine if the DNA sequences required for recognition of $P_{u r e A}$ by $H p N i k R$ are the same in vitro and in vivo. To this end, we constructed transcriptional reporters in which the wildtype ureA promoter or mutant versions of the promoter were fused to the gene encoding GFP. Reporter assays were then used to monitor ureA promoter activity directly in $H$. pylori. The wildtype $P_{\text {ureA }}$ sequence exhibited high levels of GFP expression that increased with increasing concentrations of nickel. Unexpectedly, mutation of the half-sites did not prevent basal level urease expression, but negated the $\mathrm{Ni}$ (II) dependence of ureA expression. These results suggested that another factor rescues $P_{\text {ureA }}$ transcription when the DNA target sequence for $H p$ NikR is compromised. Herein, we show that the HpArsRS acid response regulatory system, which has previously been shown to regulate ure $A$ expression in response to low $\mathrm{pH}$, also affects $u$ reA transcription at neutral $\mathrm{pH}$. This is the first time that $H p A r s R$ has been shown to regulate urease expression in conjunction with $H p \mathrm{NikR}$, and we propose that there is a cooperative interaction between these two regulators to control urease expression in $H$ pylori.

\section{Materials and Methods}

\section{Bacterial Strains and Growth}

Primer sequences are listed in Table $\mathbf{1}$ and bacterial strains and plasmids used in this study are listed in Table 2. The H. pylori strains used in this study are all derivatives of G27 (Covacci et al., 1993; Baltrus et al., 2009). H. pylori strains were maintained as frozen stocks at $-80^{\circ} \mathrm{C}$ in brain heart infusion broth (BD Biosciences) supplemented with $10 \%$ fetal bovine serum (Gibco) and 20\% glycerol (EMD chemicals, Inc.). Bacterial strains were cultured essentially as previously described (Carpenter et al., 2007). Briefly, strains were grown on horse blood agar (HBA) plates that contained $4 \%$ Columbia agar base (Neogen Corporation), 5\% defibrinated horse blood (HemoStat Laboratories, Dixon, CA), $0.2 \% \beta$ cyclodextrin (Sigma), $10 \mu \mathrm{g} / \mathrm{ml}$ vancomycin (Amresco), $5 \mu \mathrm{g} / \mathrm{ml}$ cefsulodin (Sigma), $2.5 \mathrm{U} / \mathrm{ml}$ polymyxin B (Sigma), $5 \mu \mathrm{g} / \mathrm{ml}$ trimethoprim (Sigma), and $8 \mu \mathrm{g} / \mathrm{ml}$ amphotericin B (Amresco). Liquid cultures of $H$. pylori were grown in brucella broth (Neogen Corporation) supplemented with $10 \%$ fetal bovine serum and $10 \mu \mathrm{g} / \mathrm{ml}$ vancomycin at $37^{\circ} \mathrm{C}$ with shaking at $100 \mathrm{rpm}$. As noted in Table 2, where appropriate, cultures and plates were supplemented with $8 \mu \mathrm{g} / \mathrm{ml}$ chloramphenicol (Cm) (EMD Chemicals, Inc.) and/or $25 \mu \mathrm{g} / \mathrm{ml}$ kanamycin (Kan) (Gibco). In addition, where detailed below, some HBA plates contained 5\% sucrose (Suc) (Sigma). Both liquid and plate cultures were grown under microaerobic conditions $\left(5 \% \mathrm{O}_{2}\right.$, $10 \% \mathrm{CO}_{2}$, and $85 \% \mathrm{~N}_{2}$ ) generated with an Anoxomat gas evacuation and replacement system (Spiral Biotech) in gas evacuation jars. Exponential phase cultures were grown in liquid culture for $20 \mathrm{~h}$, while stationary phase cultures were grown for $44 \mathrm{~h}$.

\section{Construction of a $\Delta$ nikR::cat $H$. pylori Strain}

The HpnikR mutant strain was constructed using a strategy that resulted in replacement of the HpnikR sequence with the cat 
TABLE 1 | List of oligonucleotides used in this study.

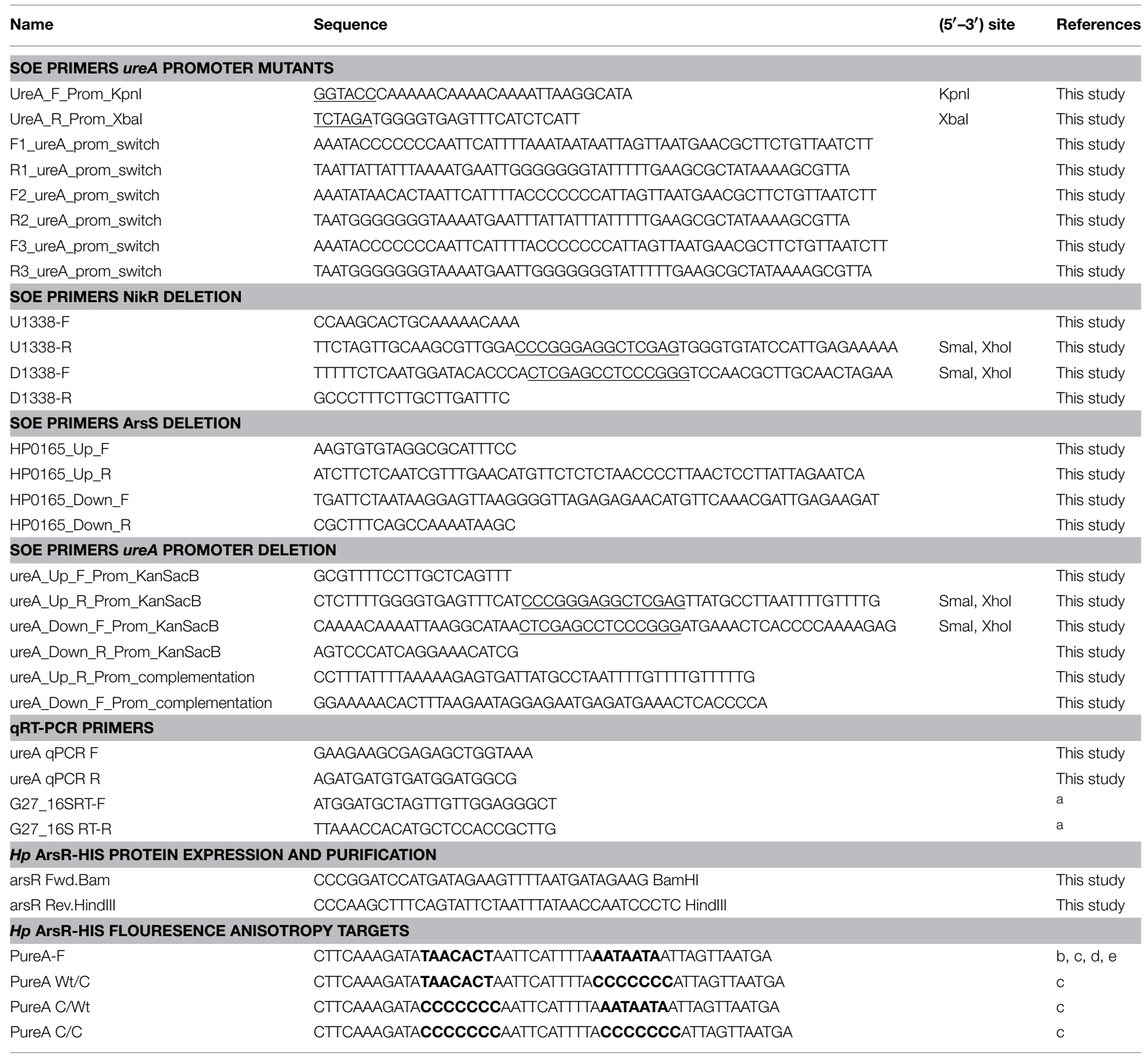

${ }^{a}$ Gillbreath et al. (2012).

${ }^{b}$ Dosanjh and Michel (2006).

cDosanih et al. (2009).

dEvans and Michel (2012).

eWest et al. (2012).

Bold print indicates the sequence within the WT ureA promoter that was chosen for mutation.

The underlined sequences correspond to the restriction sites for the enzymes listed in the same row.

gene, which encodes for chloramphenicol resistance. Briefly, a 968 bp fragment containing the region directly upstream of $n i k R$ was amplified with primers U1338-F and U1338-R, the latter of which was engineered to contain SmaI and XhoI restriction sites. This fragment was then fused via splicing by overlap extension (SOE) PCR to a $1098 \mathrm{bp}$ fragment containing the region immediately downstream of $n i k R$, which was amplified with primers D1338-F and D1338-R, the former of which contains SmaI and XhoI restriction sites. The SOE product was cloned into pGEM-T Easy to create pDSM923. Sequence analysis showed that only the SmaI site was preserved in the SOE fusion product. The cat gene (Carpenter et al., 2007), which had first been cloned into pGEM-T Easy as pDSM278, was liberated via restriction digestion with EcoR1 New England Biolabs 
TABLE 2 | List of strains used in this study.

\begin{tabular}{|c|c|c|}
\hline Plasmid or Strain & Description & References \\
\hline \multicolumn{3}{|l|}{ PLASMIDS } \\
\hline pDSM278 & pGEM T-easy::cat & This study \\
\hline pDSM462 & pGEM T-easy::WT ureA promoter & This study \\
\hline pDSM923 & pGEM T-easy::upstream region of nikR fused to downstream region & This study \\
\hline pDSM924 & pGEM T-easy:: $\Delta$ nikR::cat & This study \\
\hline pDSM922 & pBluescript:: $\Delta$ arsS::kan-sacB & This study \\
\hline pDSM1070 & pGEM T-easy::upstream region of arsS fused to downstream region & This Study \\
\hline pDSM199 & pTM117::promoterless & Carpenter et al., 2007 \\
\hline pDSM463 & pTM117::WT ureA promoter & This study \\
\hline pDSM697 & pTM117:: ureA promoter mutant C/C & This study \\
\hline pDSM698 & pTM117:: ureA promoter mutant C/WT & This study \\
\hline pDSM796 & pTM117:: ureA promoter mutant WT/C This study & \\
\hline \multicolumn{3}{|l|}{ STRAINS } \\
\hline \multicolumn{3}{|l|}{ H. pylori strains } \\
\hline G27 & WT & Baltrus et al., 2009 \\
\hline DSM215 & G27 (pTM117::promoterless), Kan $^{r}$ & This study \\
\hline DSM464 & G27 (pTM117::WT ureA promoter), Kan $^{r}$ & This study \\
\hline DSM763 & G27 (pTM117:: ureA promoter mutant C/C), Kan ${ }^{r}$ & This study \\
\hline DSM764 & G27 (pTM117:: ureA promoter mutant C/WT), Kan ${ }^{r}$ & This study \\
\hline DSM797 & G27 (pTM117:: ureA promoter mutant WT/C), $\mathrm{Kan}^{\mathrm{r}}$ & This study \\
\hline DSM975 & G27 $\Delta$ nikR::cat, $\mathrm{Cm} \mathrm{r}$ & This study \\
\hline DSM980 & G27 snikR::cat (pTM117::promoterless), $\mathrm{Cm}^{r}$, Kan ${ }^{r}$ & This study \\
\hline DSM976 & G27 $\Delta$ nikR::cat (pTM117::Wt ureA promoter), $\mathrm{Cm}^{r}, \mathrm{Kan}^{\mathrm{r}}$ & This study \\
\hline DSM977 & G27 snikR::cat (pTM117:: ureA promoter mutant C/C), $\mathrm{Cm}^{r}, \mathrm{Kan}^{r}$ & This study \\
\hline DSM978 & G27 snikR::cat (pTM117:: ureA promoter mutant CMT), $\mathrm{Cm}^{\mathrm{r}}, \mathrm{Kan}^{\mathrm{r}}$ & This study \\
\hline DSM979 & G27 $\Delta$ nikR::cat (pTM117:: ureA promoter mutant WT/C), $\mathrm{Cm}^{r}, \mathrm{Kan}^{r}$ & This study \\
\hline DSM983 & G27 $\Delta$ arsS markerless & This study \\
\hline DSM1069 & G27 $\Delta$ arsS::kan-sacB, Kan ${ }^{r}$, Suc $^{s}$ & This study \\
\hline DSM1071 & G27 $\Delta$ arsS/nikR::cat, $\mathrm{Cm}^{r}$ & This study \\
\hline DSM1398 & G27 $\Delta$ arsS markerless (pTM117::promoterless), Kan ${ }^{r}$ & This study \\
\hline DSM1399 & G27 $\Delta$ arsS markerless (pTM117::WT ureA promoter), Kan ${ }^{r}$ & This study \\
\hline DSM1400 & G27 $\Delta$ arsS markerless (pTM117:: ureA promoter mutant C/C), Kan ${ }^{r}$ & This study \\
\hline DSM1401 & G27 $\Delta$ arsS markerless (pTM117:: ureA promoter mutant CNT), Kan ${ }^{r}$ & This study \\
\hline DSM1402 & G27 $\Delta$ arsS markerless (pTM117:: ureA promoter mutant WT/C), Kan ${ }^{r}$ & This study \\
\hline DSM1403 & G27 $\Delta$ arsS/nikR::cat (pTM117::promoterless), Cmr, Kan ${ }^{r}$ & This study \\
\hline DSM1404 & G27 $\Delta$ arsS/nikR::cat (pTM117::WT ureA promoter), $\mathrm{Cm}^{r}, \mathrm{Kan}^{r}$ & This study \\
\hline DSM1405 & G27 $\Delta$ arsS/nikR::cat (pTM117:: ureA promoter mutant C/C), $\mathrm{Cm}^{\mathrm{r}}, \mathrm{Kan}^{\mathrm{r}}$ & This study \\
\hline DSM1406 & G27 $\Delta$ arsS/nikR::cat (pTM117:: ureA promoter mutant C/WT), $\mathrm{Cm}^{r}, \mathrm{Kan}^{r}$ & This study \\
\hline DSM1407 & G27 $\Delta$ arsS/nikR::cat (pTM117:: ureA promoter mutant WT/C), $\mathrm{Cm}^{r}, \mathrm{Kan}^{r}$ & This study \\
\hline
\end{tabular}

(NEB), Klenow (NEB) treated, and then ligated with the SmaI (NEB) digested and calf intestine phosphatase (NEB) treated, pDSM923; the resulting nikR deletion construct was named pDSM924. pDSM924 was subsequently transformed into G27, and transformants were selected for on HBA plates containing $8 \mu \mathrm{g} / \mathrm{mL} \mathrm{Cm}$. Resulting colonies were screened for differences in size in the nikR region via PCR with the U1338-F and D1338-R primers. For those colonies showing the expected change in size, the PCR product was next sequenced to verify deletion of nikR. One such colony showing a deletion insertion of $n i k R$ was named DSM975.

\section{Construction of a Markerless $\Delta$ arsS H. pylori Strain}

The arsS mutant strain was created using pDSM922, which is a suicide vector that contains a counter selectable marker (generous gift of Liz Marcus and David Scott, UCLA School of Medicine). Briefly, the plasmid contains a kan-sacB counter selectable cassette, previously described by Copass et al. (1997), that is flanked by the 600 and $400 \mathrm{bp}$ immediately upstream and downstream, respectively, of HP0165 (arsS). These regions were originally amplified using strain 43,504 as the template (Marshall and Goodwin, 1987). pDSM922 was naturally transformed into 
G27, and transformants were selected on HBA plates containing $25 \mu \mathrm{g} / \mathrm{ml}$ kanamycin. The resulting transformants were patched on HBA plates containing 5\% sucrose to ensure sucrose sensitivity, and deletion insertion of ars $S$ was then confirmed by PCR amplification of the region with HP0165_up_F and HP0165_down_R primers followed by sequencing with the same primers. One such $\Delta$ ars $S$ mutant was named DSM1069, which then served as the background strain to create the markerless mutant.

To create the markerless deletion strain, the region immediately upstream and downstream of ars $S$ were fused together via SOE PCR; the upstream region was amplified with the HP0165_Up_F and HP0165_Up_R primers, the downstream region was amplified with the HP0165_Down_F and HP0165_Down_R primers, and the products from these reactions were gel purified and fused together via SOE PCR using primers (HP0165_Up_F and HP0165_Down_R). The resulting product was gel purified, and naturally transformed into DSM1069. Transformants were selected on HBA plates containing 5\% sucrose and then patched onto HBA plates containing $25 \mu \mathrm{g} / \mathrm{ml}$ kanamycin to ensure kanamycin sensitivity; double crossover homologous recombination resulted in the replacement of the kan-sacB cassette to create a markerless deletion of the ars $S$ gene. Proper integration was confirmed by PCR and sequencing with the HP0165_Up_F and HP0165_Down_R primers. The resulting strain was named DSM983.

\section{Construction of a $\Delta$ arsS/nikR::cat $H$. pylori Strain}

The $\Delta a r s S / n i k R:: c a t$ mutant strain was created by naturally transforming pDSM924, the nikR::cat deletion construct, into H. pylori strain DSM983. Transformants were then screened on HBA plates supplemented with $8 \mu \mathrm{g} / \mathrm{mL} \mathrm{Cm}$ to ensure chloramphenicol resistance. Proper integration was confirmed first by PCR with the U_1338_F and D_1338_R primers. For those colonies showing the expected change in size for the nikR gene, the PCR product was sequenced to verify deletion of $n i k R$. To ensure that the $\Delta$ ars $S$ markerless deletion was still intact for this strain, PCR with the HP0165_Up_F and HP0165_Down_R primers followed by sequencing of the PCR product was performed. The resulting strain was named DSM1071.

\section{Construction of ureA::GFP Transcriptional Fusions}

Transcriptional fusions to the promoterless $g f p m u t 3$ allele carried on pTM117 (Carpenter et al., 2007) were constructed to monitor ureA expression. Briefly, the wildtype ureA promoter was PCR amplified from G27 using the UreA_F_Prom_KpnI and UreA_R_Prom_XbaI primers; these primers incorporate KpnI and XbaI restriction sites, respectively. The resulting PCR fragment was subcloned into pGEM-T Easy (Promega) to create pDSM462. The ureA promoter was then removed via digestion with KpnI (NEB) and XbaI (NEB) and ligated into the appropriately digested pTM117 vector to create pDSM463. The proper fusion was confirmed by PCR amplification with the UreA_F_Prom_KpnI and UreA_R_Prom_XbaI primers and by sequencing with the same primers. pDSM463 was then naturally transformed into G27, DSM975, DSM983 and DSM1071, and transformants were selected on HBA plates containing $25 \mu \mathrm{g} / \mathrm{ml}$ Kan. The individual strains transformed with pDSM463 are described in Table 2.

Mutant ureA promoter constructs were each created using SOE and primer pairs that incorporated the desired mutation during the process of amplification. Specifically, the C/WT mutant promoter was created using the primer pairs F1_ureA_prom_switch and UreA_R_Prom_XbaI, and UreA_F_Prom_KpnI and R1_ureA_prom_switch. The WT/C mutant promoter was created using the primer pairs F2_ureA_prom_switch and UreA_R_Prom_XbaI, and UreA_F_Prom_KpnI and R2_ureA_prom_switch. Finally, the $\mathrm{C} / \mathrm{C}$ ureA promoter mutant was created using the primer pairs F3_ureA_prom_switch and UreA_R_Prom_XbaI, and UreA_F_Prom_KpnI and R3_ureA_prom_switch. Each of the mutant ureA promoters were subcloned into pGEM-T Easy (Promega), removed by digestion with KpnI (NEB) and XbaI (NEB), and ligated into the appropriately digested pTM117 vector. The constructed plasmids are pDSM697 (C/C), pDSM698 (C/WT), and pDSM796 (WT/C). All fusions were confirmed by PCR amplification with UreA_F_Prom_KpnI and UreA_R_Prom_XbaI primers and by sequencing with the same primers. Each of the resulting plasmids containing the individual ureA promoter mutations as well as a promoterless GFP fusion vector (pDSM199) were naturally transformed into G27, DSM975, DSM983, and DSM1071, and transformants were selected on HBA plates containing $8 \mu \mathrm{g} / \mathrm{ml} \mathrm{Cm}$ and $25 \mu \mathrm{g} / \mathrm{ml}$ Kan (DSM 975 and DSM 1071), or $25 \mu \mathrm{g} / \mathrm{ml}$ Kan alone (G27 and DSM 983).

\section{GFP Reporter Assays}

The ability of the ureA transcriptional fusions to drive expression of GFP was assessed visually utilizing an Olympus BX61 fluorescent microscope, as well as using flow cytometry as previously described (Carpenter et al., 2007). Briefly, strains containing the ureA promoter fusions were grown overnight in liquid cultures containing varying $\mathrm{NiSO}_{4}$ concentrations $(0,0.5$, $1.0,10 \mu \mathrm{M})$ (Sigma). Following overnight growth, $0.5-1.5 \mathrm{ml}$ of each culture was pelleted and resuspended in $1-2 \mathrm{ml}$ of sterile $1 \times$ phosphate-buffered saline depending on the density of the culture. Bacterial clumps and culture debris were subsequently removed by passing the resuspended culture through a $1.2-\mu \mathrm{m}$ Acrodisc PSF syringe filter (Pall). Flow cytometry analysis for the ureA fusion constructs was performed using either a Beckman Coulter Epics XL-MCL flow cytometer with a laser setting of $750 \mathrm{~V}$ or a BD SLR II flow cytometer. 20,000 events were collected for each assay. WinList 3D, version 6.0 (Verity Software House) and FlowJo, version X (FLOWJO, LLC) were used to analyze the flow cytometry data. These experiments were performed 3-5 times for each strain-reporter plasmid combination.

\section{RNA Isolation, cDNA Synthesis and RT-PCR}

In addition to the GFP reporter assays, RT-PCR was utilized to measure ureA expression under normal, $10 \mu \mathrm{M}$ nickel supplemented, low $\mathrm{pH}$ ( $\mathrm{pH}$ 5.0), and combined nickel supplementation and low $\mathrm{pH}$ conditions. Bacterial liquid 
cultures of DSM1 (WT G27), DSM975 ( $\Delta$ nikR), DSM983 $(\triangle a r s S)$, and DSM1071 ( $\Delta$ arsS/nikR) were grown for $18 \mathrm{~h}$, and then each culture was divided into four equal portions. The first portion was utilized for RNA isolation and represents the normal media sample. The remaining portions were pelleted and resuspended in one of the following supplemented liquid culture medias: $10 \mu \mathrm{M} \mathrm{NiSO}_{4}$ (Sigma-Aldrich), pH 5.0 (achieved through the addition of $\mathrm{HCl}$ to the media), or $\mathrm{pH} 5.0$ with $10 \mu \mathrm{M}$ $\mathrm{NiSO}_{4}$. These portions of each culture were then maintained for another 90 min prior to RNA isolation. RNA isolation was performed as previously described (Thompson et al., 2003). The integrity of the RNA was determined through visualization on agarose gels. Next, cDNA was generated as previously described (Gilbreath et al., 2012) using the Quantitect reverse transcriptase kit (Qiagen) according to the manufacturer's protocol. Control reactions for each sample were also performed without the addition of reverse transcriptase (noRT) enzyme. Following cDNA synthesis, quantitative real-time PCR (qPCR) for ureA as well as the $16 \mathrm{~S}$ internal reference gene was performed using the primers listed in Table 1. qPCR was conducted similar to the methods used by Gilbreath et al. (2012). Briefly, qPCR reaction mixtures composed of $1 \mathrm{x}$ Roto-Gene SYBR green RT-PCR master mix, 3 pmol each of forward and reverse primer pair, and $1 \mu \mathrm{L}$ of either cDNA or noRT reaction for use as template were combined and brought to a total volume of $20 \mu \mathrm{L}$ with water. The following 2-step cycling conditions were used: $5 \mathrm{~min}$ at $95^{\circ} \mathrm{C}$ (initial activation) followed by 35 cycles of $5 \mathrm{~s}$ at $95^{\circ} \mathrm{C}$ (denaturation) and $10 \mathrm{~s}$ at $50^{\circ} \mathrm{C}$ (annealing/extension); SYBR green fluorescence was measured at each extension step. Relative gene expression was calculated using the $2^{-\Delta \Delta C T}$ method. Postrun melt curve analysis were performed to ensure specificity of amplification. Four biologically independent replicates of these experiments were conducted.

\section{H. pylori HpArsR Cloning, Expression and Purification}

PCR primers were designed to amplify the arsR gene from $H$. pylori $\mathrm{J99}$ with BamH1 and HindIII restriction sites included. The arsR gene was ligated into a $\mathrm{pQE} 3$ vector (Qiagen), which includes an $\mathrm{N}$-terminus hexa-histidine coding sequence. The identity of the cloned arsR gene was confirmed by DNA sequencing. For protein expression, arsR-pQE3 was transformed into E. coli M15-pREP4 cells, and grown in LB medium containing ampicillin $(100 \mu \mathrm{g} / \mathrm{mL})$ and kanamycin $(20 \mu \mathrm{g} / \mathrm{mL})$. When the culture reached an $A_{600}$ of 0.6 , protein expression was induced for $4 \mathrm{~h}$ with $1 \mathrm{mM}$ isopropyl- $\beta$-d-thiogalactopyranoside (IPTG). Cells were collected by centrifugation at $12,000 \times \mathrm{g}$ for $10 \mathrm{~min}$. All buffers utilized in the HpArsR studies contained $5 \mathrm{mM}$ tris(2-carboxyethyl) phosphine hydrochloride (TCEP) to prevent oligomerization of the protein. Cell pellets were resuspended in $20 \mathrm{mM}$ Tris- $\mathrm{HCl}, 500 \mathrm{mM} \mathrm{NaCl}, 5 \mathrm{mM}$ TCEP, $1 \mathrm{mM}$ phenylmethylsulfonyl fluoride (PMSF) $\mathrm{pH} 7.5$ and then lysed by sonication on ice. After sonication, the preparation was centrifuged at $31,000 \times \mathrm{g}$ for $20 \mathrm{~min}$. The supernatant $(\sim 100 \mathrm{ml})$ was collected and applied to a $30-\mathrm{ml}$ metal affinity chromatography column (His-Bind, Novagen) charged with $\mathrm{Ni}(\mathrm{II})$. The column was washed with a $50 \mathrm{mM}$ imidazole gradient to remove proteins bound non-specifically to the column, and the protein of interest, including the hexa-histidine tag, was eluted with $250 \mathrm{mM}$ imidazole over $45 \mathrm{ml}$. Five milliliter fractions corresponding to the elution were collected, and the purity of the proteins was visually assessed using 15\% SDS-PAGE gels stained with coomasie. Fractions determined to be $>95 \%$ pure were pooled, and buffer exchanged into $50 \mathrm{mM}$ Tris- $\mathrm{HCl}, 5 \mathrm{mM}$ $\mathrm{MgCl}_{2}, 50 \mathrm{mM} \mathrm{KCl}, 5 \mathrm{mM}$ TCEP, pH 7.5 and concentrated to a volume of $6 \mathrm{ml}$ using Amicon Ultra-15 centrifugal filters [5-kDa molecular weight cut-off (MWCO) membrane] for use in DNA binding assays.

\section{In vitro Phosphorylation of HpArsR}

$H p A r s R$ was phosphorylated (HpArsR-P) in vitro by incubating the protein in phosphorylation buffer $(50 \mathrm{mM}$ Tris- $\mathrm{HCl}$, $5 \mathrm{mM} \mathrm{MgCl} 2,50 \mathrm{mM} \mathrm{KCl}, 5 \mathrm{mM}$ TCEP, pH 7.5) with $50 \mathrm{mM}$ acetylphosphate for $60 \mathrm{~min}$ at $25^{\circ} \mathrm{C}$ (McCleary and Stock, 1994; Dietz et al., 2002). Phosphorylation yields were not independently measured. Post-phosphorylation, the $\mathrm{KCl}$ concentration was adjusted to $100 \mathrm{mM}$ to be consistent with past salt concentrations used in the studies of $H p$ NikR DNA recognition (Dosanjh et al., 2007, 2009; West et al., 2012).

\section{Oligonucleotide Probes}

HPLC-purified oligonucleotide probes were purchased from Integrated DNA Technologies (Coralville, IA) and were either labeled with fluorescein (F) or unlabeled as indicated in Table 1. Upon receipt, the probes were resuspended in DNasefree water and quantified. To obtain double stranded probes, each oligonucleotide probe was mixed with a probe with the complementary sequence such that there was a 1.25:1 ratio of unlabeled to labeled oligonucleotide probe in annealing buffer $(10 \mathrm{mM}$ Tris, $10 \mathrm{mM} \mathrm{NaCl}$ at $\mathrm{pH} 8.0)$. The annealing reaction mixtures were placed in a water bath set to a temperature $10^{\circ} \mathrm{C}$ higher than the melting temperatures (Tms) of the component oligonucleotides. The water bath was then immediately turned off, and the annealing reaction mixtures were allowed to cool overnight. Double-stranded oligonucleotides were quantified and stored at $-20^{\circ} \mathrm{C}$ (Dosanjh et al., 2007, 2009; West et al., 2012).

\section{Fluorescence Anisotropy Monitored Titrations of HpArsR-P and HpArsR with $\mathrm{P}_{\text {ureA }}$}

A fluorescence anisotropy (FA) assay was used to characterize the interaction of HpArsR-P and HpArsR with the ureA promoter and related mutants. Measurements were taken on an ISS PC-1 spectrofluorimeter configured in the $\mathrm{L}$ format with an excitation wavelength of $495 \mathrm{~nm}$ and an emission wavelength of $519 \mathrm{~nm}$. The band-pass for excitation was $2 \mathrm{~nm}$ and $1 \mathrm{~nm}$ for emission. $15 \mathrm{nM}$ of $P_{\text {ure }}$ in $50 \mathrm{mM}$ Tris- $\mathrm{HCl}, 5 \mathrm{mM} \mathrm{MgCl} 2,100 \mathrm{mM} \mathrm{KCl}$, $5 \mathrm{mM}$ TCEP, pH 7.5 was added to a cuvette that had been pretreated with $5 \mathrm{mM}$ bovine serum albumin (BSA) to prevent adherence of either the protein or DNA to the cuvette walls.

Direct titrations: For direct titrations, either HpArsR-P or $H p$ ArsR was titrated into fluorescein labeled ureA (ureA-F) and the change in anisotropy as a function of added protein was measured. The data were analyzed by converting the anisotropy to fraction bound, $\mathrm{F}_{\text {bound }}$ (the fraction of HpArsR-P bound 
to DNA at a given DNA concentration), using the equation (Lakowicz, 1999):

$$
F_{\text {bound }}=\frac{r-r_{\text {free }}}{\left(r_{\text {bound }}-r\right) Q+\left(r-r_{\text {free }}\right)}
$$

Where $r_{\text {free }}$ is the anisotropy of the fluorescein-labeled oligonucleotide, $r_{\text {bound }}$ is the anisotropy of the DNA/protein complex at saturation, and $\mathrm{Q}$, is the quantum yield ratio of the bound to the free form and is calculated from the fluorescence intensity changes that occur $\left(Q=I_{\text {bound }} / I_{\text {free }}\right)$. The typical $\mathrm{Q}$ for $H p$ ArsR-P DNA binding experiments was $Q=0.87$. F $F_{\text {bound }}$ was plotted against the protein concentration and fit using a one-site binding model:

$$
\begin{gathered}
P+D \rightleftharpoons P D \\
K_{d}=\frac{[P][D]}{[P D]} \\
F_{\text {bound }}=\frac{P_{\text {total }}+D_{\text {total }}+K_{d}-\sqrt{\left(P_{\text {total }}+D_{\text {total }}+K_{d}\right)^{2}-4 P_{\text {total }} D_{\text {total }}}}{2 D_{\text {total }}}
\end{gathered}
$$

Where $\mathrm{P}$ is the protein concentration and $\mathrm{D}$ is the DNA concentration. Each data point from the FA assay represents the average of 31 readings taken over a time course of $100 \mathrm{~s}$. Each titration was carried out in triplicate.

\section{Competitive Titrations}

For competitive titrations, an unlabeled DNA oligomer was titrated into a solution containing $1500 \mathrm{nM} H p A r s R-$ $\mathrm{P}$ and $5 \mathrm{nM} P_{\text {ureA }}-F$ and the decrease in anisotropy (r) as the unlabeled DNA oligomer competed with the labeled oligonucleotide was recorded. The resultant anisotropy values were converted to fraction bound. The competition experiments were performed with HpArsR-P concentrations at levels near saturation to minimize the amount of unlabeled DNA required to complete the titrations. Experiments were performed aerobically as no difference in binding was observed between experiments performed anaerobically and aerobically (data not shown).

Binding isotherms were fit using Mathematica (version 8 Wolfram Research) to a model that involved the mass action equations for the three competing equilibria:

$$
\begin{gathered}
P+D_{f} \underset{K_{1}}{\overleftrightarrow{\longrightarrow}} P D_{f} \\
P+D \underset{K_{2}}{\overleftrightarrow{\longrightarrow}} P D \\
P D_{f}+D \underset{K_{3}}{\longleftrightarrow} P D+D_{f}
\end{gathered}
$$

Where $\mathrm{P}$ is the protein (HpArsR-P), $\mathrm{D}_{f}$ is fluorescently labeled DNA, and D represents unlabeled DNA. The value for $K_{1}$ was determined from the forward titrations and thus used as a known parameter for the fit. Mathematica software was used to combine Equations (1)-(3) and to solve the resulting cubic equation in terms of $P D_{f}$ using non-linear, least squares analysis. All titrations were carried out in triplicate (Dosanjh et al., 2009).

\section{Statistical Analysis}

Statistical analysis on qRT-PCR and flow cytometry data was conducted using GraphPad Prism 6.01 (GraphPad Software, La Jolla, CA, USA). A One-Way ANOVA was used to compare basal levels of expression in $\Delta n i k R, \Delta a r s S$, and $\Delta$ arsS/nikR to WT. A Two-Way ANOVA with Tukey's correction for multiple comparisons was used to analyze fold differences in expression

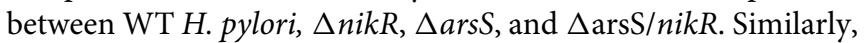
mean fluorescent intensity (MFI) values obtained from flow cytometry were assessed using a two-way ANOVA with Tukey's correction for multiple comparisons. A $p<0.05$ was considered significant.

\section{Results}

\section{Regulation of $\boldsymbol{P}_{\text {ureA }}$ by $\mathrm{HpNikR}$}

$H p$ NikR positively regulates the expression of urease by binding to a partially palindromic sequence located on the ure $A$ promoter ( $\left.P_{\text {ureA }}\right)$ (Dosanjh et al., 2007, 2009; Evans and Michel, 2012). The DNA sequence that $H p N i k R$ recognizes and binds to is AT rich and consists of seven base pairs separated by an eleven base pair linker sequence that is not thought to be directly involved in the protein/DNA recognition event (Figures 1B,C). When either half of the palindrome is mutated to all cytosines, the in vitro affinity of $H p \mathrm{NikR}$ for the DNA sequence is diminished by 3 orders of magnitude from a $\mathrm{K}_{d}$ of $8.0 \pm 1 \mathrm{nM}$ to a $\mathrm{K}_{d}$ of $1.0 \pm 0.09$ or $4.9 \pm 0.8 \mu \mathrm{M}$ for the $\mathrm{WT} / \mathrm{C}$ or $\mathrm{C} / \mathrm{WT}$ mutants, respectively (Dosanjh et al., 2009; West et al., 2012). When both halves of the palindrome are mutated to all cytosines, DNA binding is fully abrogated (Dosanjh et al., 2009). Thus, both sides of the recognition palindrome appear to be important for $H p$ NikR/DNA binding.

To determine if these in vitro identified DNA sequence requirements are also observed in vivo, a series of Green Fluorescent Protein (GFP) reporter constructs in which the DNA recognition sequence within the ureA promoter was varied, to mirror the modifications that were studied in vitro, were created. These constructs, named WT/WT, WT/C, C/WT, and $\mathrm{C} / \mathrm{C}$, were unmodified or modified at each palindrome as indicated in Table 2. To measure the effect of these modifications on the nickel-dependent transcription of urease controlled by endogenous $H p \mathrm{NikR}$, expression of GFP was monitored in $H$. pylori strain G27 and an isogenic $\triangle n i k R$ strain grown in the presence of increasing $\mathrm{Ni}$ (II) concentrations.

Visual inspection of the strains by fluorescence microscopy showed that fluorescence was only observed in strains carrying constructs in which the ureA promoter was fused to GFP; promoterless controls showed no fluorescence (data not shown). Quantitative analysis of GFP expression was achieved using flow cytometry, as described in the materials and methods. Maximum GFP expression was observed for the WT/WT reporter construct carried in the wildtype $H$. pylori strain. This was the only reporter construct for which $\mathrm{Ni}$ (II) dependence was observed; a statistically significant concentration-dependent increase in GFP expression was observed as the $\mathrm{Ni}$ (II) concentration was increased (Figure 2 and Tables 3, 4). In the wildtype H. pylori strain background, GFP expression levels for the WT/C and C/C 


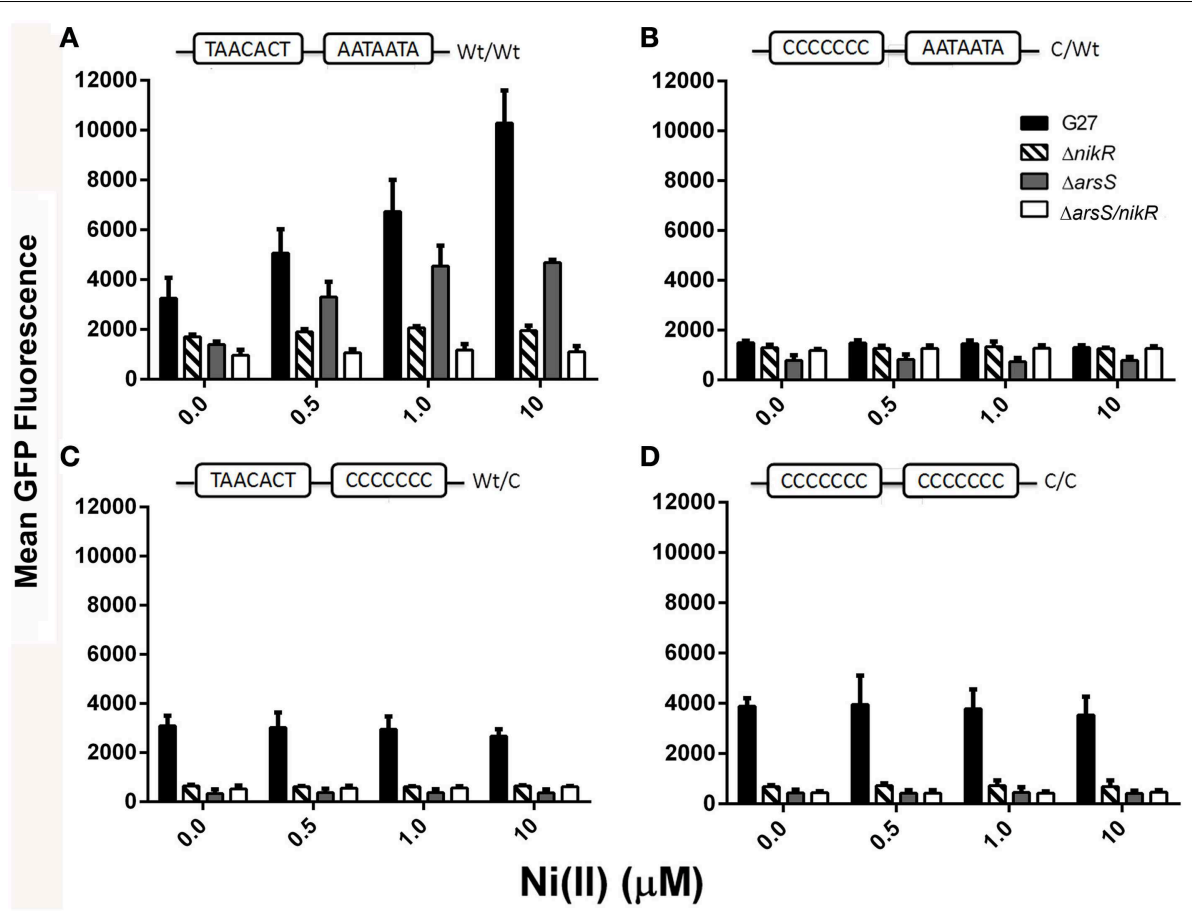

FIGURE 2 | Regulation of $\boldsymbol{P}_{\text {ure }} \boldsymbol{A}$ by $\boldsymbol{H p N i k R}$ and $\boldsymbol{H p A r s R S . ~ T h e ~ m e a n ~}$ GFP expression for the $P_{\text {ureA }}$ WT and mutant constructs expressed in the various parental strains is shown. Each panel corresponds to a different $\mathrm{P}_{\text {ureA }}$ mutant construct that is indicated at the top of each panel: (A)WTMT, (B)- CNT, (C)- WT/C, and (D)- C/C. Shading is as indicated: WT
G27 (black bars), $\Delta$ nikR (cross bars), $\Delta$ arsS (gray bars), and $\Delta a r s S / n i k R$ (white bars) in $\mathrm{NiSO}_{4}$ supplemented $(0,0.5,1,10 \mu \mathrm{M})$ growth media. Flow cytometry was performed 3-5 times for each strain-reporter plasmid combination. Bars represent the mean GFP fluorescence and the error bars indicate the standard deviation. reporter constructs were similar to the GFP expression levels observed for the WT/WT reporter construct when the media was not supplemented with $\mathrm{Ni}$ (II) (Figure 2 and Table 4). In contrast, basal levels of ure A expression in the C/WT background were significantly lower than WT/WT in the wildtype $H$. pylori strain $(p<0.0001)$. At high Ni (II) concentrations, the reporter constructs containing mutation of the palindrome half sites (WT/C and C/WT) as well as full site (C/C) exhibited decreased GFP expression levels when compared to the unmodified WT/WT construct (Table 4). En masse, the fact that we observed high basal levels of expression of ureA with some of the mutant constructs was unexpected; our in vitro findings suggest that mutation of both sides of the palindrome completely abrogate $H p$ NikR binding at the ureA promoter (Dosanjh et al., 2009). Given that nickel responsiveness was completely lost for each mutated promoter construct (Figure 2 and Table 4) and because increasing nickel concentrations are known to result in increased $H p N i k R$ activity, this finding suggested that another regulator may play a role in ureA expression under the in vivo conditions examined in these experiments.

To specifically examine the contribution of $H p \mathrm{NikR}$ to the observed levels of ureA expression, the same four promoter constructs were next examined in an isogenic $\Delta n i k R$ strain. Interestingly, GFP expression was not entirely abrogated for the WT/WT promoter though expression was not nickel responsive (Figure 2 and Table 4). Once again, this suggests that the GFP expression observed in the wildtype strain is regulated by $H p N i k R$ as well as another regulatory factor (Figure 2). Mutating either side of the $H p \mathrm{NikR}$ binding sequence in the $P_{\text {ure }}$ promoter abrogated the $\mathrm{Ni}$ (II) response and resulted in varying effects on basal levels of ureA expression. Mutation of the right half of the recognition sequence $(\mathrm{WT} / \mathrm{C})$ resulted in a significant decrease in expression $(p=0.0021)$ as compared to the WT/C construct in the wildtype strain. Likewise, disruption of both segments of the palindrome $(\mathrm{C} / \mathrm{C})$ resulted in a dramatic decrease in ureA expression in the $\Delta n i k R$ mutant (Figure 2) that was similar to the decrease observed in the WT/C construct (Table 4). Interestingly, mutating the left half $(\mathrm{C} / \mathrm{WT})$ of the recognition sequence did not lead to a significant reduction in ureA expression $(p=0.4408)$ as compared to the C/WT construct in the wildtype strain. When considered together, these findings suggest that an additional regulatory factor is involved in urease transcription. Moreover, given that in vitro studies have clearly shown that $H p \mathrm{NikR}$ does not bind to the C/C mutant construct (Dosanjh et al., 2009), the demonstrated decrease in transcription when $H p \mathrm{NikR}$ is absent in the strain carrying the $\mathrm{C} / \mathrm{C}$ mutant construct suggests that $H p$ NikR has a hitherto unidentified indirect role in $P_{\text {ureA }}$ transcription.

\section{Regulation of $\boldsymbol{P}_{\text {ureA }}$ by $\boldsymbol{H p A r s R}$}

The observation that $P_{\text {ure }}$ transcription occurs for the WT/C and C/WT GFP reporter constructs in the presence and absence 
TABLE 3 | Mean GFP fluorescence in normal and $10 \mu \mathrm{M} \mathrm{NiSO}{ }_{4}$ supplemented media.

\begin{tabular}{|c|c|c|c|c|c|c|c|c|c|c|c|c|}
\hline \multirow[t]{2}{*}{ Strain } & \multicolumn{3}{|c|}{ WT/WT } & \multicolumn{3}{|c|}{ WT/C } & \multicolumn{3}{|c|}{ C/WT } & \multicolumn{3}{|c|}{ C/C } \\
\hline & N. media* & $+\mathrm{Ni}(\mathrm{II})^{\dagger}$ & $\begin{array}{c}\mathrm{Ni}(\mathrm{II}) \\
\text { dependent }\end{array}$ & N. media* & $+\mathrm{Ni}(\mathrm{II})^{\dagger}$ & $\begin{array}{c}\mathrm{Ni}(\mathrm{II}) \\
\text { dependent }\end{array}$ & N. media* & $+\mathrm{Ni}(\mathrm{II})^{\dagger}$ & $\begin{array}{c}\mathrm{Ni}(\mathrm{II}) \\
\text { dependent }\end{array}$ & N. media* & $+\mathbf{N i}(\mathrm{II})^{\dagger}$ & $\begin{array}{c}\mathrm{Ni}(\mathrm{II}) \\
\text { dependent }\end{array}$ \\
\hline G27 & 3252 & 10282 & yes & 3094 & 2671 & no & 1498 & 1309 & no & 3890 & 3538 & no \\
\hline$\Delta n i k R$ & 1708 & 1959 & no & 642 & 643 & no & 1298 & 1259 & no & 686 & 686 & no \\
\hline$\Delta$ arsS & 1399 & 4688 & yes & 332 & 368 & no & 782 & 785 & no & 439 & 410 & no \\
\hline$\Delta a r s S / n i k R$ & 963 & 1110 & no & 527 & 618 & no & 1186 & 1266 & no & 446 & 465 & no \\
\hline
\end{tabular}

* All values are represented as mean GFP fluorescence.

$t_{10} \mathrm{MM} \mathrm{NiSO}_{4}$ added to media.

of $H p \mathrm{NikR}$ independent of nickel availability suggested that another factor regulates $u r e A$ transcription in these conditions. The two-component system HpArsRS has been shown to mediate $\mathrm{pH}$-responsive urease expression (Pflock et al., 2005, 2006a,b; Wen et al., 2006, 2007). Furthermore, the HpArsR binding site within the ureA promoter partially overlaps the site recognized by $H p N i k R$ (Figures 1B,C). To determine if $H p A r s R$ was responsible for the observed urease expression, $\Delta$ ars $S$ and $\triangle a r s S / n i k R$ deletion strains of $H$. pylori were constructed and GFP fluorescence was measured for each of the $P_{\text {ureA }}$ constructs. Of note, this strategy was chosen since $H p A r s R$ was previously shown to be essential and cannot be deleted (Beier and Frank, 2000; McDaniel et al., 2001); however, HpArsR regulates urease in its phosphorylated form (HpArsR-P) (Pflock et al., 2005). Therefore, deletion of $H p$ ArsS effectively inactivates $H p A r s R$ dependent regulation of urease since $H p A r s S$ is required for HpArsR phosphorylation (Pflock et al., 2005, 2006a).

To dissect the role of $H p A r s R(\triangle a r s S)$ in ureA expression, transcription was measured for each of the $P_{\text {ure }}$ constructs. As expected, because $H p \mathrm{NikR}$ is present in the $\Delta a r s S$ strain background, the WT/WT promoter showed $\mathrm{Ni}$ (II) dependent GFP expression. However, the relative amounts of GFP expression were significantly lower in this strain background as compared to the wildtype strain (Table 4): for example, a mean GFP fluorescence of 4688 fluorescence units was measured for the $\Delta$ ars $S$ strain in $10 \mu \mathrm{M}$ nickel as compared to 10,282 fluorescence units for wildtype under the same nickel concentrations (Figure 2 and Table 3). This finding suggests that under non-acidic conditions, $H p A$ ArsR-P interacts cooperatively with $H p$ NikR to increase expression from the WT urease promoter. In the $\triangle a r s S / n i k R$ background, though a basal level of GFP expression is observed, this expression is nickel independent (Figure 2).

For the WT/C and $\mathrm{C} / \mathrm{C}$ mutant promoters, the absence of endogenous $H p A$ ArsS (and thus, $H p A$ rsR-P) resulted in lower levels of GFP expression as compared to those observed in the wildtype strains ( $p<0.0001$ for both). In each case, GFP expression was unaffected by nickel concentration. Similar to what was seen in the $\Delta n i k R$ background, while the WT/C and $\mathrm{C} / \mathrm{C}$ promoters resulted in decreased expression, the decrease in ureA expression in the C/WT background was not significant as compared to the same construct carried in the wildtype strain (Table 4). Furthermore, basal levels of ureA expression in $\triangle n i k R$ and $\triangle a r s S$ strains were similar regardless of which ureA promoter was present (Table 4). In the $\Delta$ ars $/$ nikR background, the WT/C and C/WT mutant promoters showed levels of expression similar to those observed in the $\Delta n i k R$ background. As was observed in the $\Delta n i k R$ single mutant background, none of these promoter constructs was nickel responsive in the double mutant background (Figure 2 and Table 3). In the $\Delta a r s S$ and $\Delta$ ars $S / n i k R$ backgrounds, the $\mathrm{C} / \mathrm{C}$ promoter fusion produced low levels of GFP expression that were slightly less than the levels observed in the $\Delta n i k R$ background. Significant GFP expression was only observed for this construct in the wildtype strain. Taken en masse, these findings are consistent with cooperative interaction of $H p \mathrm{NikR}$ and $H p A r s R-P$ at the ureA promoter to achieve maximal regulation of urease. Of note, the role of HpArsR-P in this regulation occurred in the absence of acidic $\mathrm{pH}$, which is considered to be the major environmental signal controlling HpArsR activity (Pflock et al., 2005, 2006a,b; Wen et al., 2006, 2007).

To further investigate the cooperative regulation of ureA by $H p N i k R$ and HpArsR-P and to confirm that the use of plasmid based transcriptional fusions was not artificially affecting our results, we next directly assessed ureA expression directly from the chromosome via qPCR analysis on RNA extracted from WT, $\triangle n i k R, \Delta a r s S$, and $\triangle \operatorname{arsS} / n i k R H$. pylori strains. We assessed ure $A$ expression in strains that were exposed to normal growth media as well as to medias that were 1) supplemented with excess nickel $(10 \mu \mathrm{M}), 2)$ adjusted to acidic $\mathrm{pH}(\mathrm{pH} 5.0)$, or 3) supplemented with excess nickel and adjusted to acidic $\mathrm{pH}$ $(10 \mu \mathrm{M}+\mathrm{pH}$ 5.0). Comparison of basal levels of ureA expression between the WT and mutant strains under normal growth conditions showed that there was little to no difference in ureA expression between WT and $\Delta n i k R(p=0.319)$; this was expected based on previous data (Ernst et al., 2005). Conversely, a statistically significant decrease in basal ureA expression was seen in the $\Delta a r s S(p=0.009)$ and $\Delta \operatorname{arsS} / n i k R(p=0.0116)$ strains (Figure 3A). As with the data obtained with the GFP fusions (Figure 2), these data suggest that $H p A r s R S$ is necessary for maximal expression of ureA under normal growth conditions.

Following exposure to $10 \mu \mathrm{M} \mathrm{Ni}^{2+}$, ureA expression was strongly upregulated in WT $H$. pylori (approximately six-fold). However, the extent of ureA upregulation was significantly decreased in the $\Delta n i k R$ strain $(p=0.0159)$. Although not significant, a decrease in $\mathrm{Ni}$-dependent upregulation of ureA was also observed in the $\Delta a r s S$ strain background (three-fold increase compared to six-fold in WT) (Figure 3B). Additionally 
TABLE 4 | Statistical analysis of mean GFP fluorescence.

WT/WT ureA promoter construct with increasing nickel concentrations

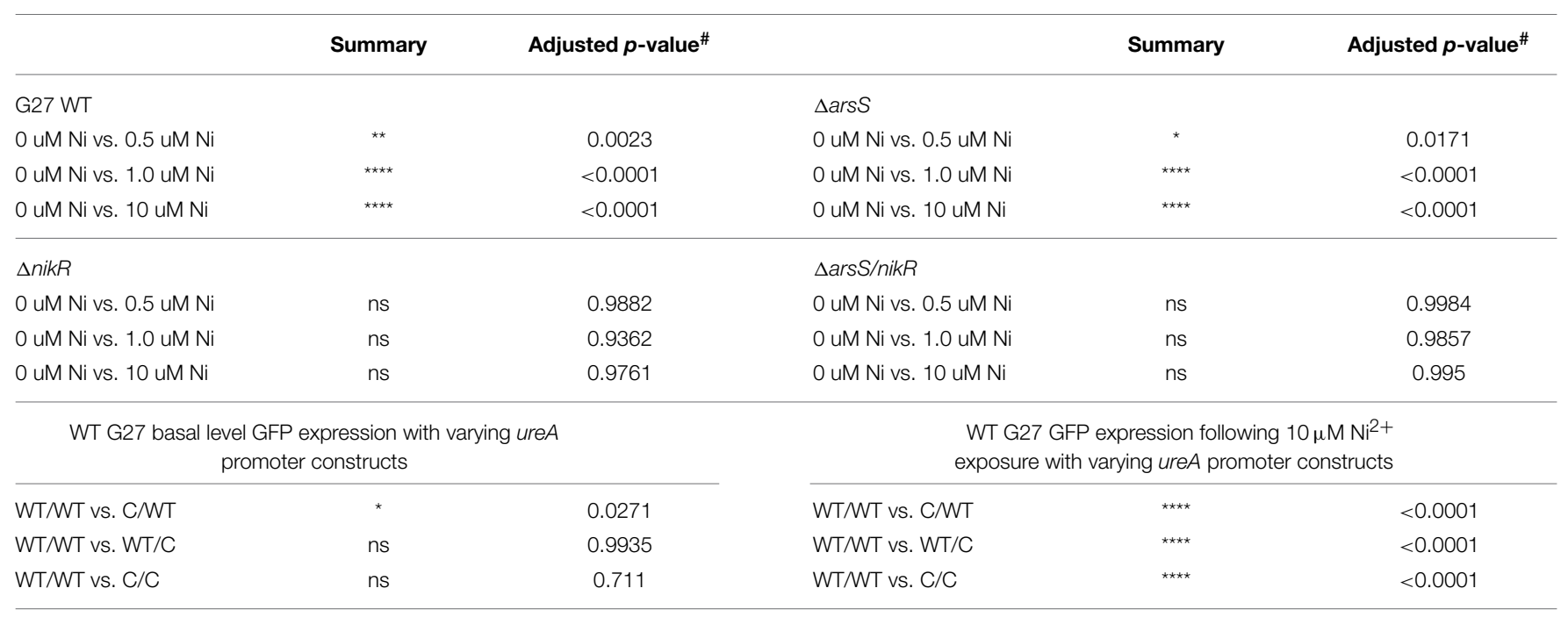

Basal level GFP expression from WT ureA promoter in varying strain backgrounds

\begin{tabular}{lll}
\hline G27 WT vs. $\Delta$ nikR & $* * * \star$ & $<0.0001$ \\
G27 WT vs. $\Delta a r s S$ & $* * * *$ & $<0.0001$ \\
G27 WT vs. $\Delta$ arsS/nikR & $* * * *$ & $<0.0001$ \\
\hline
\end{tabular}

Basal GFP expression

\section{C/WT}

G27 WT vs. $\Delta$ nikR

G27 WT vs. $\Delta$ arsS

G27 WT vs. $\Delta$ arsS/nikR

ns $\quad 0.8802$

ns $\quad 0.0567$

ns $\quad 0.6586$

WT/C

G27 WT vs. $\Delta$ nikR

G27 WT vs. $\Delta a r s S$

G27 WT vs. $\Delta a r s S / n i k R$

$\begin{array}{ll}* \star \star \star & <0.0001 \\ * \star * \star & <0.0001 \\ & <0.0001\end{array}$

$\mathrm{C} / \mathrm{C}$

G27 WT vs. $\Delta$ nikR

G27 WT vs. $\Delta a r s S$

G27 WT vs. $\Delta a r s S / n i k R$

$\begin{array}{ll}* * * * & <0.0001 \\ * * * * & <0.0001 \\ * * * * & <0.0001\end{array}$

GFP expression following $10 \mu \mathrm{M} \mathrm{Ni}^{2+}$ exposure from WT ureA promoter in varying strain backgrounds

\begin{tabular}{lll}
\hline G27 WT vs. $\Delta$ nikR & $* * * *$ & $<0.0001$ \\
G27 WT vs. $\Delta$ arsS & $* \star \star *$ & $<0.0001$ \\
G27 WT vs. $\Delta$ arsS/nikR & $* * * *$ & $<0.0001$
\end{tabular}

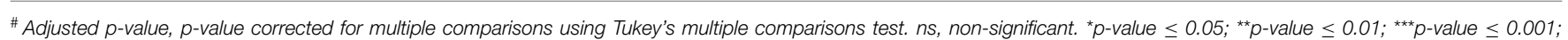
${ }^{\star * \star \star} p$-value $<0.0001$

in the $\Delta a r s S / n i k R$ double mutant there was no change in ureA expression upon exposure to nickel (Figure 3B). These data suggest that both $H p \mathrm{NikR}$ and $H p A r s R S$ are required for maximal expression of ureA upon nickel stress. This point is further supported by the fact that the observed difference in nickel dependent ureA expression between $\Delta n i k R$ and $\triangle a r s S$ was not statistically significant $(p=0.5529)$. Similarly, upon exposure to acidic $\mathrm{pH}$, ureA expression was increased approximately eightfold in the WT strain, six-fold in $\triangle n i k R$, five-fold in $\Delta a r s S$ but only two-fold in the $\triangle a r s S / n i k R$ double mutant (Figure 3C). A statistically significant difference in $u r e A$ expression under low $\mathrm{pH}$ was only observed when comparing WT and the
$\Delta$ arsS/nikR double mutant $(p=0.0467)$. Given that similar levels of ure $A$ expression were observed in both the $\Delta n i k R$ and $\Delta$ ars $S$ strains, this suggests that both regulatory proteins are necessary for maximal expression in the low $\mathrm{pH}$ environment; thus, $H p N i k R$ appears to play a previously unknown role in the acid responsive regulation of ureA (Figure 3C). Lastly, changes in $u r e A$ expression were monitored following simultaneous exposure to excess nickel and low $\mathrm{pH}$. Again, the largest increase in expression was observed in the WT strain background (ninefold). Although not statistically significant, ureA expression was only moderately increased in the $\triangle n i k R$ (four-fold) and $\triangle a r s S$ (three-fold) strains (Figure 3D). Of note, under these conditions, 


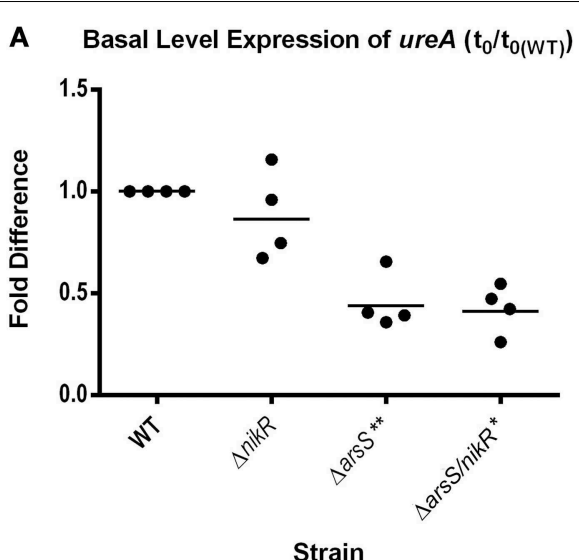

C

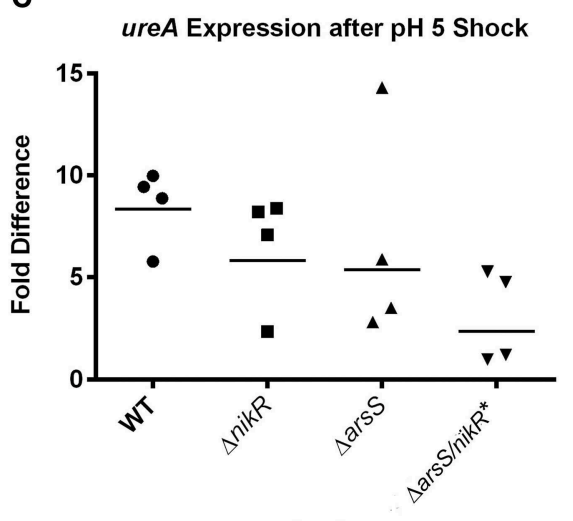

Strain

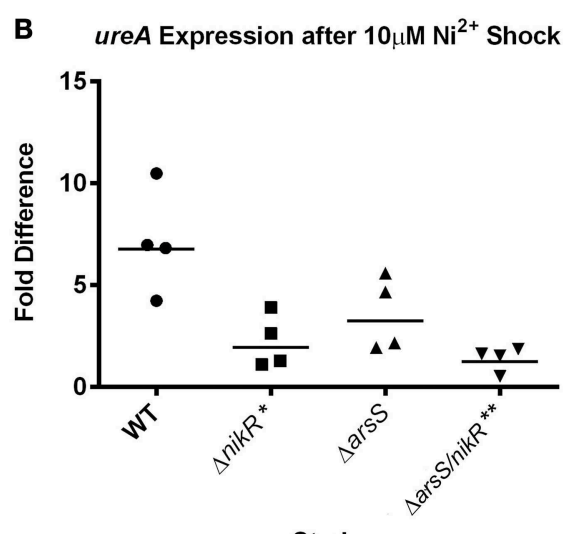

Strain

D

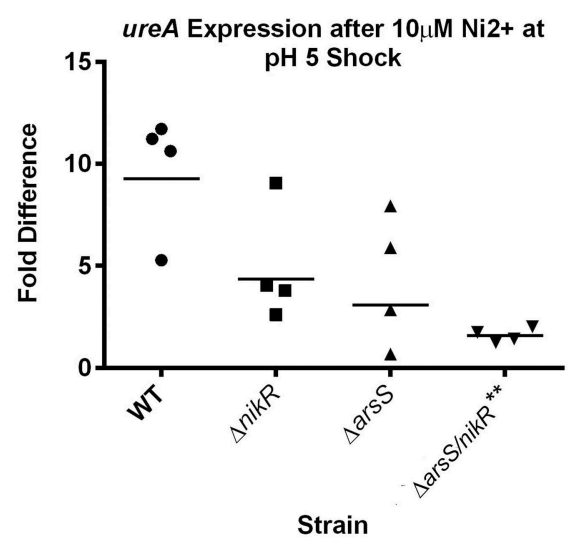

expression following shock with $10 \mu \mathrm{M} \mathrm{Ni}^{2+}$; (C) Changes in ureA expression following shock with $\mathrm{pH}$ 5.0; (D) Changes in ureA expression following shock with $10 \mu \mathrm{M} \mathrm{Ni}^{2+}$ at $\mathrm{pH}$ 5.0. Four biologically independent replicates of these experiments were conducted, and each dot represents the fold difference from one replicate with bars representing the geometric mean fold difference. ${ }^{\star} p<0.05$ compared to $\mathrm{WT},{ }^{* *} p<0.01$ compared to $\mathrm{WT}$. $\triangle n i k R$ and $\triangle a r s S$ were not significantly different from each other ( $p=0.8729)$ or from the $\Delta$ ars $S / n i k R$ double mutant $(p=0.1722$ and $p=0.4847$, respectively). However, given that no change in $u$ reA expression was observed for the $\triangle a r s S / n i k R$ double mutant, ureA expression in the $\triangle$ ars $S$ nikR double mutant was significantly different from WT ( $p=0.0059$ ) (Figure 3D). En masse, these data support the notion that both $H p N i k R$ and $H p A r s R S$ are important for regulation of ureA expression under normal conditions as well as in low $\mathrm{pH}$ and nickel supplemented environments.

\section{Fluorescence Anisotropy to Measure HpArsR-P/P $\boldsymbol{P}_{\text {ure } A}$ Binding in vitro}

Based on previous DNase footprinting studies, HpArsR-P binds to two regions of the $P_{\text {ureA }}$ promoter (Pflock et al., 2005). These two operators are made up of 41 and 57 base pairs, respectively, and the larger operator sequence includes bases recognized by $H p N i k R$ (Figures 1B,C). Based upon our in vivo results that suggest co-regulation of the ureA promoter by $H p A r s R$ and
$H p \mathrm{NikR}$, the in vitro DNA binding properties of $H p A$ rsR-P for the $P_{\text {ure }}$ sequences recognized by $H p N i k R$ were measured using fluorescence anisotropy (FA), which is an approach that has been successfully used to measure $H p \mathrm{NikR} / \mathrm{P}_{\text {ureA }}$ binding (Dosanjh et al., 2007, 2009; Evans and Michel, 2012; West et al., 2012). The FA data for $H p A$ rsR-P with $P_{\text {ureA }}$ WT/WT, WT/C, C/WT, and $\mathrm{C} / \mathrm{C}$ showed comparable binding isotherms (Figure 4), with $\mathrm{K}_{d} \mathrm{~s}$ of $17 \pm 2.0,24 \pm 0.5,20 \pm 0.6$, and $23 \pm 0.7 \mathrm{nM}$ respectively. These findings show that $H p$ ArsR-P can bind to the same $P_{\text {ureA }}$ promoter sequence as $H p N i k R$ in vitro, and it can also bind to sequences in which the palindrome recognized by $H p N i k R$ is altered. HpArsR did not exhibit any DNA binding (data not shown).

\section{Discussion}

$H p$ NikR regulates urease production by binding to and activating transcription of $P_{\text {ureA }}$ (van Vliet et al., 2002, 2004a; Abraham et al., 2006; Dosanjh et al., 2007). The details of the protein/DNA 


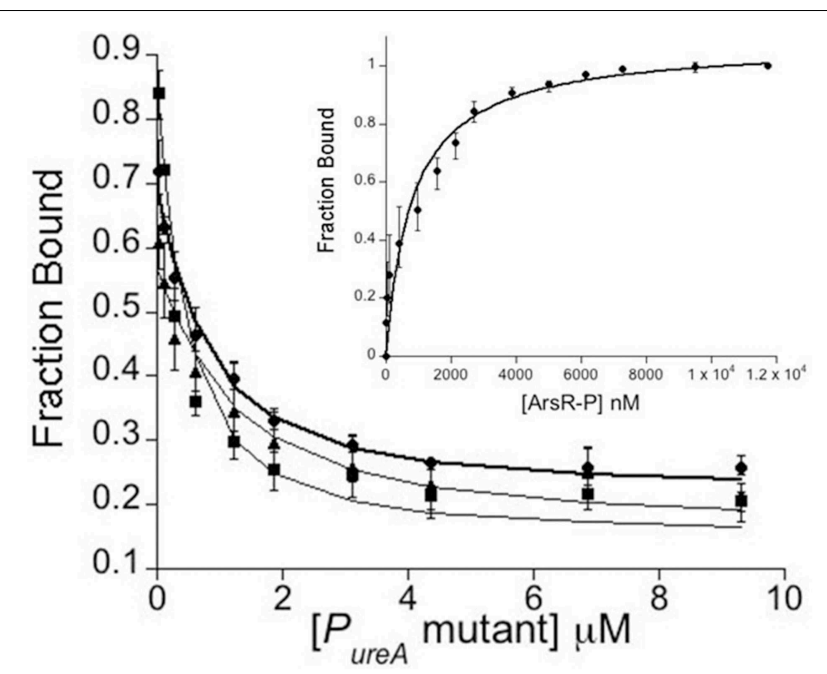

FIGURE 4 | Inset Direct fluorescence anisotropy (FA) titration between HpArsR-P and fluorescein labeled $\mathbf{W t} / \mathbf{W t} \boldsymbol{P}_{\text {ure }} \boldsymbol{A}-\boldsymbol{F}$. The data are fit to a 1:1 binding equilibrium. Main Figure: competitive titrations of HpArsR-P with PureA mutants: $\bullet P_{\text {ureA }} W t / C, \square P_{\text {ureA }} \mathrm{C} / \mathrm{Wt}$, and $\Delta P_{\text {ureA }} \mathrm{C} / \mathrm{C}$ into $5 \mathrm{nM}$ $\mathrm{P}_{\text {ureA }}-\mathrm{F}$ and $1.5 \mu \mathrm{M}$ HpArsR-P. The data are fit to a competitive binding equilibrium. The data shown are the average of three sets of binding data. All FA experiments were performed in $50 \mathrm{mM}$ Tris- $\mathrm{HCl}, 5 \mathrm{mM} \mathrm{MgCl} 2,100 \mathrm{mM}$ $\mathrm{KCl}, 5 \mathrm{mM}$ TCEP, $\mathrm{pH} 7.5$ and $25^{\circ} \mathrm{C}$.

binding interaction have been systematically studied in vitro and a key partially palindromic recognition sequence has been identified (van Vliet et al., 2002, 2004a; Abraham et al., 2006; Dosanjh et al., 2007). When either half-site of the recognition palindrome is modified to all cytosines, the affinity of $H p N i k R$ for the DNA is significantly reduced, while mutation of both half sites to all cytosines abrogates binding (Dosanjh et al., 2009). Those findings led us to conclude that these half-sites were the recognition elements required for $H p N i k R$-mediated activation of ureA expression. Herein, we present studies designed to translate our previous in vitro findings to the in vivo conditions present within the $H$. pylori cell. These in vivo findings revealed that additional factors are involved in the regulation of urease. Specifically, maximal activation of ureA transcription required the $H p A$ ArsRS two component system in addition to $H p N i k$.

The initial suggestion that a second factor may regulate ureA transcription came from GFP reporter assays of the $P_{\text {ureA }} \mathrm{WT} / \mathrm{C}$ and C/WT half-site mutants. Some level of nickel-independent GFP expression was still observed for all $P_{\text {ure }}$ constructs; though, the levels of expression were only nickel dependent in WT/WT (Figure 2 and Table 4). Interestingly, while $\mathrm{P}_{\text {ure }}$ transcription was significantly decreased for the C/WT half site mutation, WT/C and C/C promoter mutations yielded similar levels of GFP expression to WT/WT in the wildtype $H$. pylori strain background (Figure 2 and Table 4). These high levels of expression suggested that a second factor was involved in regulating ureA transcription in vivo. This hypothesis was further supported by the levels of ureA expression observed in the $\Delta n i k R$ strain background. For the $P_{\text {ureA }}$ half-site mutant, C/WT, the level of ure $A$ transcription was similar to that observed in the wildtype strain while for the WT/C mutant, the level of ureA transcription was approximately five-times less than in the wildtype strain. Additionally, expression of the $\mathrm{C} / \mathrm{C}$ mutant construct was greatly diminished (approximately six times), indicating that the high level of expression seen for this construct in the wildtype strain background was $H p N i k R$ dependent. However, in vitro protein/DNA binding data has clearly demonstrated that $H p N i k R$ is unable to physically interact with this altered C/C DNA target. Thus, the role that $H p N i k R$ plays in regulating the $\mathrm{C} / \mathrm{C}$ target appears to be indirect.

Given these data, we sought to identify the other factor required for proper regulation of ureA transcription at neutral $\mathrm{pH}$ (pH 7.5). The HpArsRS two-component system is also known to control urease expression in H. pylori. However, this system, which is responsive to $\mathrm{pH}$, is thought to function primarily under acidic conditions (Marcus et al., 2012). The data presented in this study, demonstrate that $H p$ ArsRS functions with $H p N i k R$ to properly regulate urease expression at neutral $\mathrm{pH}$. Within the HpArsRS two-component system, $H p$ ArsS functions as the histidine kinase, and $H p A r s R$ functions as the cognate response regulator. Upon sensing acidic $\mathrm{pH}, \mathrm{HpArsS}$ phosphorylates $H p A r s R$, which results in activation of the regulator (Pflock et al., 2006a; Joseph and Beier, 2007; Gupta et al., 2009; Muller et al., 2009). Interestingly, HpArsS is not essential for $H$. pylori survival, but $H p A r s R$ is essential (Schar et al., 2005). This suggests that the non-phosphorylated form of HpArsR regulates some essential component within the H. pylori cell. HpArsR has been shown to bind some target promoters in its nonphosphorylated form, while it only binds other promoters in its phosphorylated form (Wen et al., 2006). The ureA promoter is known to be bound and regulated by the phosphorylated form of HpArsR (Pflock et al., 2005). Thus, deletion of arsS from G27 results in an inactive $H p A$ rsR response regulator in terms of regulation of the ureA promoter. Using this strain we found that although expression of the WT/WT fusion was still nickel-dependent, the expression levels were significantly lower at all Ni (II) concentrations tested than in the G27 background (Figure 2 and Table 4), suggesting a role for $H p A r s R-P$ in maximal expression from this promoter. Similarly, the WT/C half-site mutant, showed considerable reduction in $\mathrm{P}_{\text {ure } A}:: g f p$ expression in the absence arsS; once again indicating a role for $H$ pArsR-P. Interestingly the C/WT half-site mutant appeared to lock the level of expression of ureA at a basal level regardless of the strain background examined. Conversely, the $\mathrm{C} / \mathrm{C}$ mutation affected ure $A$ transcription in all of the tested mutant strain backgrounds, suggesting concomitant regulation by both $H p N i k R$ and $H p$ ArsRS. Additionally, qPCR analysis revealed that basal levels of ureA expression were significantly reduced in the absence of $\operatorname{ars} S(p=0.009)$ but not nikR ( $p=$ 0.319 ) (Figure 3A). Further support for this idea comes from the analysis of $u r e A$ expression following exposure to nickel, low $\mathrm{pH}$ or both stressors combined. Regardless of the stressor, the highest levels of ureA expression were observed in the WT strain background, with each single deletion showing only moderate levels of ure $A$ and the least amount of expression occurring in the double $\Delta$ arsS/nikR strain (Figure 3). 
Taken together, these data support a model of ureA transcription that involves "cross-talk" between $H p N i k R$ and $H p A r s R S$ to maximize induction of urease even under neutral conditions. Prior to this study, these two regulators were believed to function independently as $H p \mathrm{NikR}$ is responsive to nickel levels and HpArsRS is responsive to acidic shock (Pflock et al., 2005). Therefore, the observation that ureA transcription is 2-2.5 times lower in the $\Delta a r s S$ background is particularly compelling since, under these conditions, $H p N i k R$ is present and functional. Surprisingly, the data presented here also indicate a role for $H p N i k R$ in the response to acid stress; previous reports suggested that the acid-induced increase in ureA expression was independent of NikR (Pflock et al., 2005). Using qualitative primer extension, Pflock et al. showed that there were similar increases in $u r e A$ expression upon exposure to $\mathrm{pH} 5.0$ in strains with and without nikR (Pflock et al., 2005). However, in this work, we observed that the increase in ureA expression was less than that of wildtype in the $\Delta n i k R$ mutant strain. The differences in our data and the previously published work could be due to differences in assay sensitivities (primer extension vs. qPCR) as well as differences in exposure to stress conditions. In the previous work, the bacteria were exposed to low $\mathrm{pH}$ for $60 \mathrm{~min}$ as compared to our $90 \mathrm{~min}$ exposure. Perhaps, a longer observation period following exposure to low $\mathrm{pH}$ allows for better measurement of the transcriptional changes in response to the stressor. Our data fully support the model that both $H p \mathrm{NikR}$ and $H p A r s R S$ are necessary for maximal levels of ureA expression in response to low $\mathrm{pH}$ regardless of nickel concentration.

Although, the discovery of cross-talk between $H p N i k R$ and $H p A r s R$ is unexpected, crosstalk, involving $H p \mathrm{NikR}, H p A r s R$, or other $H$. pylori regulatory proteins in general, is not unique. For example, $H p$ NikR and $H p F u r$ co-regulate fur transcription (Delany et al., 2001, 2005) and HpArsS and HpFlgS work in concert to recruit and activate urease (Marcus et al., 2012). In addition, regulatory crosstalk among transcription factors has been observed within E. coli. Specifically, the transcription factors MarA and Rob of E. coli, which are involved in the response to chemical stressors consequently enabling antibiotic resistance, are co-regulated through transcriptional cross-talk with each other (Miller et al., 1994; Martin et al., 1996; Martin and Rosner, 1997; Michan et al., 2002; Schneiders and Levy, 2006; McMurry and Levy, 2010; Warner and Levy, 2010).

Though the in vivo transcriptional assays revealed a role of $H p A r s R S$ in regulation of ureA transcription, they did not demonstrate whether this role was a direct protein/DNA binding interaction, or an indirect effect via another, yet to be identified, factor. As previous studies have shown a requirement of phosphorylation for DNA binding at the ureA promoter (Pflock et al., 2005), a direct effect would likely involve HpArsR-P (Dietz et al., 2002). Using FA, we examined whether HpArsR-P directly bound to the four DNA targets (WT/WT, WT/C, C/WT, and $\mathrm{C} / \mathrm{C}$ ) by titrating $\mathrm{H} p$ ArsR-P with fluorescently tagged DNA targets. A change in FA, indicative of binding, was observed for all four DNA targets when HpArsR-P was titrated. No DNA binding was observed when the control non-phosphorylated HpArsR was studied. These data indicate that $H p A r s R S$ directly regulates ure $A$ by binding to a 48 -mer promoter sequence.
Together, the in vitro and in vivo data obtained for HpArsR$\mathrm{P}$ provide valuable insight into the role of $H p A r s R$ from a biophysical and a biological perspective. The in vitro results that we obtained for $H p A$ ArsR-P binding to ureA (and related mutants) teach us that $H p A$ ArsR-P binds to the ure $A$ promoter in a very different way than $H p \mathrm{NikR}$. $H p \mathrm{NikR}$ requires a specific sequence (the pseudo-palindrome) for high affinity DNA binding (Dosanjh et al., 2009; Evans and Michel, 2012). In contrast, HpArsR$\mathrm{P}$ does not require this specific pseudo-palindromic sequence for binding (i.e., there is equivalent binding when the pseudopalindromic sequence is modified).

Two factors are often important when proteins bind to DNA: sequence and shape (Rohs et al., 2010; Parker and Tullius, 2011). For $H p \mathrm{NikR}$ evidence indicates that sequence - the pseudopalindromic sequence found within the ureA promoter - is important for binding; when the sequence is modified, binding is abrogated (Dosanjh et al., 2009; Evans and Michel, 2012). In contrast, for HpArsR-P the data indicate that the pseudopalindromic sequence is not important; when the sequence is modified, binding is not affected (vide supra). This may mean that HpArsR-P/ureA binding is driven by shape (overall conformation/structure of the DNA), rather than sequence, or that the sequence recognized by $H p A r s R-P$ contains additional oligonucleotides than the sequence recognized by $H p N i k R$.

The in vivo data, for which the entire promoter is present (rather than the short stretch of DNA utilized in the in vitro binding studies), revealed that when the ureA sequence was modified to the C/WT sequence and HpArsR-P driven expression was measured, the expression decreased. In contrast, $H p A$ ArsR-P driven expression for all of the other ureA sequences was not dramatically affected. This finding indicates that there must be another factor (or factors), beyond the direct recognition of HpArsR-P with the 48-mer ureA target sequences in vitro, that is important for HpArsR-P regulation of ureA in vivo.

The combination of in vitro and in vivo data presented here allows us to learn both about (i) the very specific binding event between HpArsR-P and ureA (48 mer), which informs on the fundamental biophysical basis of binding, and (ii) the overall regulation by $H p$ ArsR-P at the cellular level, which informs on the biological mechanism. We initiated these studies to determine how the in vitro $H p N i k R / u r e A$ binding that we had previously measured translated in an in vivo setting. The data indicate that the in vivo regulation is more complex that the in vitro protein/DNA binding. Furthermore, we identified HpArsR-P as another key player in this regulation. By then looking at HpArsR-P both in vivo and in vitro, we can draw the same conclusion regarding $H p A$ ArsR-P function: that its role in regulation in vivo is more complex than its in vitro protein/DNA binding.

Prior to the work presented here, $H p N i k R$ and $H p A r s R S$ were thought to function as independent regulators of transcription, with $H p N i k R$ involved in regulation of urease in response to intracellular nickel availability and $H p$ ArsRS involved in urease regulation in response to intracellular acid shock (Pflock et al., 2005). Strikingly, the studies presented here reveal that both $H p$ NikR and HpArsR-P are necessary for maximum $\mathrm{Ni}(\mathrm{II})$ dependent regulation of urease in vivo as well as the maximal 
response to acid shock. The two proteins are not independent regulators but, instead, work cooperatively to regulate ureA transcription. This is the first time that "cross-talk" between $H p N i k R$ and HpArsRS has been demonstrated, and further studies will be required to tease out the interactions that promote this cooperative effect.

\section{Author Contributions}

Conceived and designed the experiments: AW, BC, DM, SM. Strain Construction: AW, BC, HG, JG. Performed the experiments: AW, HG, BC, DH, OP, SS. Contributed reagents/materials/analysis tools: $\mathrm{MF}, \mathrm{DH}$. Wrote the paper: $\mathrm{AW}$, BC, DM, SM.

\section{References}

Abraham, L. O., Li, Y., and Zamble, D. B. (2006). The metal- and DNA-binding activities of Helicobacter pylori NikR. J. Inorg. Biochem. 100, 1005-1014. doi: 10.1016/j.jinorgbio.2005.10.014

Bahlawane, C., Dian, C., Muller, C., Round, A., Fauquant, C., Schauer, K., et al. (2010). Structural and mechanistic insights into Helicobacter pylori NikR activation. Nucleic Acids Res. 38, 3106-3118. doi: 10.1093/nar/gkp1216

Baltrus, D. A., Amieva, M. R., Covacci, A., Lowe, T. M., Merrell, D. S., Ottemann, K. M., et al. (2009). The complete genome sequence of Helicobacter pylori strain G27. J. Bacteriol. 191, 447-448. doi: 10.1128/JB.01416-08

Beier, D., and Frank, R. (2000). Molecular characterization of twocomponent systems of Helicobacter pylori. J. Bacteriol. 182, 2068-2076. doi: 10.1128/JB.182.8.2068-2076.2000

Benanti, E. L., and Chivers, P. T. (2007). The N-terminal arm of the Helicobacter pylori $\mathrm{Ni}^{2+}$-dependent transcription factor NikR is required for specific DNA binding. J. Biol. Chem. 282, 20365-20375. doi: 10.1074/jbc.M702982200

Benanti, E. L., and Chivers, P. T. (2011). Helicobacter pylori NikR protein exhibits distinct conformations when bound to different promoters. J. Biol. Chem. 286, 15728-15737. doi: 10.1074/jbc.M110.196055

Benini, S., Cianci, M., and Ciurli, S. (2011). Holo-Ni ${ }^{2+}$ Helicobacter pylori NikR contains four square-planar nickel-binding sites at physiological pH. Dalton Trans. 40, 7831-7833. doi: 10.1039/c1dt11107h

Blaser, M. J. (1990). Helicobacter pylori and the pathogenesis of gastroduodenal inflammation. J. Infect. Dis. 161, 626-633. doi: 10.1093/infdis/161.4.626

Borin, B. N., Tang, W., and Krezel, A. M. (2014). Helicobacter pylori RNA polymerase alpha-subunit C-terminal domain shows features unique to varepsilon-proteobacteria and binds NikR/DNA complexes. Protein Sci. 23, 454-463. doi: 10.1002/pro.2427

Carpenter, B. M., McDaniel, T. K., Whitmire, J. M., Gancz, H., Guidotti, S., Censini, S., et al. (2007). Expanding the Helicobacter pylori genetic toolbox: modification of an endogenous plasmid for use as a transcriptional reporter and complementation vector. Appl. Environ. Microbiol. 73, 7506-7514. doi: 10.1128/AEM.01084-07

Carter, E. L., Flugga, N., Boer, J. L., Mulrooney, S. B., and Hausinger, R. P. (2009). Interplay of metal ions and urease. Metallomics 1, 207-221. doi: 10.1039/b903311d

Chivers, P. T., and Sauer, R. T. (1999). NikR is a ribbon-helix-helix DNA-binding protein. Protein Sci. 8, 2494-2500. doi: 10.1110/ps.8.11.2494

Chivers, P. T., and Sauer, R. T. (2000). Regulation of high affinity nickel uptake in bacteria. $\mathrm{Ni}^{2+}$-Dependent interaction of $\mathrm{NikR}$ with wild-type and mutant operator sites. J. Biol. Chem. 275, 19735-19741. doi: 10.1074/jbc.M002232200

Chivers, P. T., and Sauer, R. T. (2002). NikR repressor: high-affinity nickel binding to the C-terminal domain regulates binding to operator DNA. Chem. Biol. 9, 1141-1148. doi: 10.1016/S1074-5521(02)00241-7

Chivers, P. T., and Tahirov, T. H. (2005). Structure of Pyrococcus horikoshii NikR: nickel sensing and implications for the regulation of DNA recognition. J. Mol. Biol. 348, 597-607. doi: 10.1016/j.jmb.2005.03.017

\section{Acknowledgments}

SLJM thanks NSF CHE1306208 for support of these studies. We are grateful to the members of the Merrell lab for their invaluable microbiology expertise. Prof. Edwin Pozharski (UMB) for assistance with PyMol, Prof. David Scott and Liz Marcus (UCLA School of Medicine) for the $\triangle$ arsS::kan-sacB deletion strain of $H$. pylori and Mohsin Khan (UMB) for editing Figure 1A. DSM thanks Prof. Andrew Snow and Sasha Larsen for their assistance with flow cytometry technique and analysis. The opinions and assertions contained herein are the private ones of the authors and are not to be construed as official or reflecting the views of the NIH, the NSF, the Department of Defense, or the Uniformed Services University of the Health Sciences.

Contreras, M., Thiberge, J. M., Mandrand-Berthelot, M. A., and Labigne, A (2003). Characterization of the roles of NikR, a nickel-responsive pleiotropic autoregulator of Helicobacter pylori. Mol. Microbiol. 49, 947-963. doi: 10.1046/j.1365-2958.2003.03621.x

Copass, M., Grandi, G., and Rappuoli, R. (1997). Introduction of unmarked mutations in the Helicobacter pylori vacA gene with a sucrose sensitivity marker. Infect. Immun. 65, 1949-1952.

Covacci, A., Censini, S., Bugnoli, M., Petracca, R., Burroni, D., Macchia, G., et al. (1993). Molecular characterization of the $128-\mathrm{kDa}$ immunodominant antigen of Helicobacter pylori associated with cytotoxicity and duodenal ulcer. Proc. Natl. Acad. Sci. U.S.A. 90, 5791-5795. doi: 10.1073/pnas.90.12.5791

Cover, T. L., and Blaser, M. J. (1992). Helicobacter pylori and gastroduodenal disease. Annu. Rev. Med. 43, 135-145. doi: 10.1146/annurev.me.43.020192.001031

Danielli, A., Romagnoli, S., Roncarati, D., Costantino, L., Delany, I., and Scarlato, V. (2009). Growth phase and metal-dependent transcriptional regulation of the fecA genes in Helicobacter pylori. J. Bacteriol. 191, 3717-3725. doi: 10.1128/JB.01741-08

Davis, G. S., Flannery, E. L., and Mobley, H. L. (2006). Helicobacter pylori HP1512 is a nickel-responsive NikR-regulated outer membrane protein. Infect. Immun. 74, 6811-6820. doi: 10.1128/IAI.01188-06

Delany, I., Ieva, R., Soragni, A., Hilleringmann, M., Rappuoli, R., and Scarlato, V. (2005). In vitro analysis of protein-operator interactions of the NikR and Fur metal-responsive regulators of coregulated genes in Helicobacter pylori. J. Bacteriol. 187, 7703-7715. doi: 10.1128/JB.187.22.7703-7715.2005

Delany, I., Spohn, G., Rappuoli, R., and Scarlato, V. (2001). The Fur repressor controls transcription of iron-activated and -repressed genes in Helicobacter pylori. Mol. Microbiol. 42, 1297-1309. doi: 10.1046/j.1365-2958.2001. 02696.x

Dietz, P., Gerlach, G., and Beier, D. (2002). Identification of target genes regulated by the two-component system HP166-HP165 of Helicobacter pylori. J. Bacteriol. 184, 350-362. doi: 10.1128/JB.184.2.350-362.2002

Dosanjh, N. S., Hammerbacher, N. A., and Michel, S. L. J. (2007). Characterization of the Helicobacter pylori NikR-P(ureA) DNA interaction: metal ion requirements and sequence specificity. Biochemistry 46, 2520-2529. doi: 10.1021/bi062092w

Dosanjh, N. S., and Michel, S. L. J. (2006). Microbial nickel metalloregulation: NikRs for nickel ions. Curr. Opin. Chem. Biol. 10, 123-130. doi: 10.1016/j.cbpa.2006.02.011

Dosanjh, N. S., West, A. L., and Michel, S. L. J. (2009). Helicobacter pylori NikR's interaction with DNA: a two-tiered mode of recognition. Biochemistry 48, 527-536. doi: 10.1021/bi801481j

Dunn, B. E., Cohen, H., and Blaser, M. J. (1997). Helicobacter pylori. Clin. Microbiol. Rev. 10, 720-741.

Ernst, F. D., Kuipers, E. J., Heijens, A., Sarwari, R., Stoof, J., Penn, C. W., et al. (2005). The nickel-responsive regulator NikR controls activation and repression of gene transcription in Helicobacter pylori. Infect. Immun. 73, 7252-7258. doi: 10.1128/IAI.73.11.7252-7258.2005 
Ernst, F. D., Stoof, J., Horrevoets, W. M., Kuipers, E. J., Kusters, J. G., and van Vliet, A. H. (2006). NikR mediates nickel-responsive transcriptional repression of the Helicobacter pylori outer membrane proteins FecA3 (HP1400) and FrpB4 (HP1512). Infect. Immun. 74, 6821-6828. doi: 10.1128/IAI.01196-06

Evans, S. E., and Michel, S. L. J. (2012). Dissecting the role of DNA sequence in Helicobacter pylori NikR/DNA recognition. Dalton Trans. 41, 7946-7951. doi: $10.1039 / \mathrm{c} 2 \mathrm{dt} 30504 \mathrm{f}$

Gilbreath, J. J., West, A. L., Pich, O. Q., Carpenter, B. M., Michel, S., and Merrell, D. S. (2012). Fur activates expression of the 2-oxoglutarate oxidoreductase genes (oorDABC) in Helicobacter pylori. J. Bacteriol. 194, 6490-6497. doi: 10.1128/JB.01226-12

Goodwin, A. C., Weinberger, D. M., Ford, C. B., Nelson, J. C., Snider, J. D., Hall, J. D., et al. (2008). Expression of the Helicobacter pylori adhesin SabA is controlled via phase variation and the ArsRS signal transduction system. Microbiology 154, 2231-2240. doi: 10.1099/mic.0.2007/016055-0

Gupta, S. S., Borin, B. N., Cover, T. L., and Krezel, A. M. (2009). Structural analysis of the DNA-binding domain of the Helicobacter pylori response regulator ArsR. J. Biol. Chem. 284, 6536-6545. doi: 10.1074/jbc.M804592200

Jones, M. D., Ademi, I., Yin, X., Gong, Y., and Zamble, D. B. (2015). Nickelresponsive regulation of two novel Helicobacter pylori NikR-targeted genes. Metallomics 7, 662-673. doi: 10.1039/c4mt00210e

Joseph, B., and Beier, D. (2007). Global analysis of two-component gene regulation in $H$. pylori by mutation analysis and transcriptional profiling. Meth. Enzymol. 423, 514-530. doi: 10.1016/s0076-6879(07)23025-3

Kusters, J. G., van Vliet, A. H., and Kuipers, E. J. (2006). Pathogenesis of Helicobacter pylori infection. Clin. Microbiol. Rev. 19, 449-490. doi: 10.1128/CMR.00054-05

Lakowicz, J. R. (1999). Principles of Fluorescence Spectroscopy . New York, NY: Kluwer Academic/Plenium Publishers.

Li, Y., and Zamble, D. B. (2009). pH-responsive DNA-binding activity of Helicobacter pylori NikR. Biochemistry 48, 2486-2496. doi: 10.1021/bi8 $01742 \mathrm{r}$

Loh, J. T., Gupta, S. S., Friedman, D. B., Krezel, A. M., and Cover, T. L. (2010). Analysis of protein expression regulated by the Helicobacter pylori ArsRS two-component signal transduction system. J. Bacteriol. 192, 2034-2043. doi: 10.1128/JB.01703-08

Loughlin, M. F. (2003). Novel therapeutic targets in Helicobacter pylori. Expert Opin. Ther. Targets 7, 725-735. doi: 10.1517/14728222.7.6.725

Maier, R. J., Benoit, S. L., and Seshadri, S. (2007). Nickel-binding and accessory proteins facilitating Ni-enzyme maturation in Helicobacter pylori. Biometals 20, 655-664. doi: 10.1007/s10534-006-9061-8

Marcus, E. A., Sachs, G., Wen, Y., Feng, J., and Scott, D. R. (2012). The role of the Helicobacter pylori sensor kinase ArsS in protein trafficking and acid acclimation. J. Bacteriol. 194, 5545-5551. doi: 10.1128/JB.01263-12

Marshall, B. J., and Goodwin, C. S. (1987). Revised nomenclature of Campylobacter pyloridis. Int. J. Syst. Bacteriol. 37, 68. doi: 10.1099/00207713-37-1-68

Marshall, B. J., and Warren, J. R. (1984). Unidentified curved bacilli in the stomach of patients with gastritis and peptic ulceration. Lancet 1, 1311-1315. doi: 10.1016/S0140-6736(84)91816-6

Martin, R. G., Jair, K. W., Wolf, R. E. Jr., and Rosner, J. L. (1996). Autoactivation of the marRAB multiple antibiotic resistance operon by the MarA transcriptional activator in Escherichia coli. J. Bacteriol. 178, 2216-2223.

Martin, R. G., and Rosner, J. L. (1997). Fis, an accessorial factor for transcriptional activation of the mar (multiple antibiotic resistance) promoter of Escherichia coli in the presence of the activator MarA, SoxS, or Rob. J. Bacteriol. 179, 7410-7419.

McCleary, W. R., and Stock, J. B. (1994). Acetyl phosphate and the activation of two-component response regulators. J. Biol. Chem. 269, 31567-31572.

McDaniel, T. K., Dewalt, K. C., Salama, N. R., and Falkow, S. (2001). New approaches for validation of lethal phenotypes and genetic reversion in Helicobacter pylori. Helicobacter 6, 15-23. doi: 10.1046/j.1523-5378.2001.00001.x

McMurry, L. M., and Levy, S. B. (2010). Evidence that regulatory protein MarA of Escherichia coli represses rob by steric hindrance. J. Bacteriol. 192, 3977-3982. doi: 10.1128/JB.00103-10

Michan, C., Manchado, M., and Pueyo, C. (2002). SoxRS down-regulation of rob transcription. J. Bacteriol. 184, 4733-4738. doi: 10.1128/JB.184.17.47334738.2002
Miller, P. F., Gambino, L. F., Sulavik, M. C., and Gracheck, S. J. (1994). Genetic relationship between soxRS and mar loci in promoting multiple antibiotic resistance in Escherichia coli. Antimicrob. Agents Chemother. 38, 1773-1779. doi: 10.1128/AAC.38.8.1773

Muller, C., Bahlawane, C., Aubert, S., Delay, C. M., Schauer, K., Michaud-Soret, I., et al. (2011). Hierarchical regulation of the NikR-mediated nickel response in Helicobacter pylori. Nucleic Acids Res. 39, 7564-7575. doi: 10.1093/nar/gkr460

Muller, S., Gotz, M., and Beier, D. (2009). Histidine residue 94 is involved in $\mathrm{pH}$ sensing by histidine kinase ArsS of Helicobacter pylori. PLoS ONE 4:e6930. doi: 10.1371/journal.pone.0006930

Parker, S. C., and Tullius, T. D. (2011). DNA shape, genetic codes, and evolution. Curr. Opin. Struct. Biol. 21, 342-347. doi: 10.1016/j.sbi.2011.03.002

Pflock, M., Finsterer, N., Joseph, B., Mollenkopf, H., Meyer, T. F., and Beier, D. (2006a). Characterization of the ArsRS regulon of Helicobacter pylori, involved in acid adaptation. J. Bacteriol. 188, 3449-3462. doi: 10.1128/JB.188.10.34493462.2006

Pflock, M., Kennard, S., Delany, I., Scarlato, V., and Beier, D. (2005). Acid-induced activation of the urease promoters is mediated directly by the ArsRS twocomponent system of Helicobacter pylori. Infect. Immun. 73, 6437-6445. doi: 10.1128/IAI.73.10.6437-6445.2005

Pflock, M., Kennard, S., Finsterer, N., and Beier, D. (2006b). Acid-responsive gene regulation in the human pathogen Helicobacter pylori. J. Biotechnol. 126, 52-60. doi: 10.1016/j.jbiotec.2006.03.045

Rohs, R., Jin, X., West, S. M., Joshi, R., Honig, B., and Mann, R. S. (2010). Origins of specificity in protein-DNA recognition. Annu. Rev. Biochem. 79, 233-269. doi: 10.1146/annurev-biochem-060408-091030

Romagnoli, S., Agriesti, F., and Scarlato, V. (2011). In vivo recognition of the fecA3 target promoter by Helicobacter pylori NikR. J. Bacteriol. 193, 1131-1141. doi: 10.1128/JB.01153-10

Sachs, G., Kraut, J. A., Wen, Y., Feng, J., and Scott, D. R. (2006). Urea transport in bacteria: acid acclimation by gastric Helicobacter spp. J. Membr. Biol. 212, 71-82. doi: 10.1007/s00232-006-0867-7

Sachs, G., Weeks, D. L., Wen, Y., Marcus, E. A., Scott, D. R., and Melchers, K. (2005). Acid acclimation by Helicobacter pylori. Physiology (Bethesda) 20, 429-438. doi: 10.1152/physiol.00032.2005

Schar, J., Sickmann, A., and Beier, D. (2005). Phosphorylation-independent activity of atypical response regulators of Helicobacter pylori. J. Bacteriol. 187, 3100-3109. doi: 10.1128/JB.187.9.3100-3109.2005

Schneiders, T., and Levy, S. B. (2006). MarA-mediated transcriptional repression of the rob promoter. J. Biol. Chem. 281, 10049-10055. doi: 10.1074/jbc.M512097200

Schreiter, E. R., and Drennan, C. L. (2007). Ribbon-helix-helix transcription factors: variations on a theme. Nat. Rev. Microbiol. 5, 710-720. doi: 10.1038/nrmicro1717

Scott, D. R., Marcus, E. A., Wen, Y., Oh, J., and Sachs, G. (2007). Gene expression in vivo shows that Helicobacter pylori colonizes an acidic niche on the gastric surface. Proc. Natl. Acad. Sci. U.S.A. 104, 7235-7240. doi: 10.1073/pnas.0702300104

Sepulveda, A. R., and Coelho, L. G. (2002). Helicobacter pylori and gastric malignancies. Helicobacter 7(Suppl. 1), 37-42. doi: 10.1046/j.1523-5378.7.s1.6.x

Stoof, J., Kuipers, E. J., and van Vliet, A. H. (2010). Characterization of NikRresponsive promoters of urease and metal transport genes of Helicobacter mustelae. Biometals 23, 145-159. doi: 10.1007/s10534-009-9275-7

Thompson, L. J., Merrell, D. S., Neilan, B. A., Mitchell, H., Lee, A., and Falkow, S. (2003). Gene expression profiling of Helicobacter pylori reveals a growth-phasedependent switch in virulence gene expression. Infect. Immun. 71, 2643-2655. doi: 10.1128/IAI.71.5.2643-2655.2003

van Vliet, A. H., Ernst, F. D., and Kusters, J. G. (2004a). NikR-mediated regulation of Helicobacter pylori acid adaptation. Trends Microbiol. 12, 489-494. doi 10.1016/j.tim.2004.09.005

van Vliet, A. H., Kuipers, E. J., Stoof, J., Poppelaars, S. W., and Kusters, J. G. (2004b). Acid-responsive gene induction of ammonia-producing enzymes in Helicobacter pylori is mediated via a metal-responsive repressor cascade. Infect. Immun. 72, 766-773. doi: 10.1128/IAI.72.2.766-773.2004

van Vliet, A. H., Poppelaars, S. W., Davies, B. J., Stoof, J., Bereswill, S., Kist, M., et al. (2002). NikR mediates nickel-responsive transcriptional induction of urease expression in Helicobacter pylori. Infect. Immun. 70, 2846-2852. doi: 10.1128/IAI.70.6.2846-2852.2002 
Warner, D. M., and Levy, S. B. (2010). Different effects of transcriptional regulators MarA, SoxS and Rob on susceptibility of Escherichia coli to cationic antimicrobial peptides (CAMPs): Rob-dependent CAMP induction of the marRAB operon. Microbiology 156, 570-578. doi: 10.1099/mic.0.033415-0

Wen, Y., Feng, J., Scott, D. R., Marcus, E. A., and Sachs, G. (2006). Involvement of the HP0165-HP0166 two-component system in expression of some acidicpH-upregulated genes of Helicobacter pylori. J. Bacteriol. 188, 1750-1761. doi: 10.1128/JB.188.5.1750-1761.2006

Wen, Y., Feng, J., Scott, D. R., Marcus, E. A., and Sachs, G. (2007). The HP0165HP0166 two-component system (ArsRS) regulates acid-induced expression of HP1186 alpha-carbonic anhydrase in Helicobacter pylori by activating the pH-dependent promoter. J. Bacteriol. 189, 2426-2434. doi: 10.1128/JB. 01492-06

Wen, Y., Feng, J., Scott, D. R., Marcus, E. A., and Sachs, G. (2009). The pHresponsive regulon of HP0244 (FlgS), the cytoplasmic histidine kinase of Helicobacter pylori. J. Bacteriol. 191, 449-460. doi: 10.1128/JB.01219-08

Wen, Y., Marcus, E. A., Matrubutham, U., Gleeson, M. A., Scott, D. R., and Sachs, G. (2003). Acid-adaptive genes of Helicobacter pylori. Infect. Immun. 71, 5921-5939. doi: 10.1128/IAI.71.10.5921-5939.2003

West, A. L., Evans, S. E., Gonzalez, J. M., Carter, L. G., Tsuruta, H., Pozharski, E., et al. (2012). Ni(II) coordination to mixed sites modulates DNA binding of HpNikR via a long-range effect. Proc. Natl. Acad. Sci. U.S.A. 109, 5633-5638. doi: $10.1073 /$ pnas. 1120283109
West, A. L., St John, F., Lopes, P. E., Mackerell, A. D. Jr., Pozharski, E., and Michel, S. L. J. (2010). Holo-Ni(II)HpNikR is an asymmetric tetramer containing two different nickel-binding sites. J. Am. Chem. Soc. 132, 14447-14456. doi: $10.1021 / \mathrm{ja} 104118 \mathrm{r}$

Wolfram, L., Haas, E., and Bauerfeind, P. (2006). Nickel represses the synthesis of the nickel permease NixA of Helicobacter pylori. J. Bacteriol. 188, 1245-1250. doi: 10.1128/JB.188.4.1245-1250.2006

Zambelli, B., Danielli, A., Romagnoli, S., Neyroz, P., Ciurli, S., and Scarlato, V. (2008). High-affinity $\mathrm{Ni}^{2+}$ binding selectively promotes binding of Helicobacter pylori NikR to its target urease promoter. J. Mol. Biol. 383, 1129-1143. doi: 10.1016/j.jmb.2008.08.066

Conflict of Interest Statement: The authors declare that the research was conducted in the absence of any commercial or financial relationships that could be construed as a potential conflict of interest.

Copyright (c) 2015 Carpenter, West, Gancz, Servetas, Pich, Gilbreath, Hallinger, Forsyth, Merrell and Michel. This is an open-access article distributed under the terms of the Creative Commons Attribution License (CC BY). The use, distribution or reproduction in other forums is permitted, provided the original author(s) or licensor are credited and that the original publication in this journal is cited, in accordance with accepted academic practice. No use, distribution or reproduction is permitted which does not comply with these terms. 